\title{
Modes of symmetric composite defects in two-dimensional photonic crystals
}

\author{
K. B. Dossou, ${ }^{*}$ C. G. Poulton, and L. C. Botten \\ Centre for Ultrahigh bandwidth Devices for Optical Systems and Department of Mathematical Sciences \\ University of Technology, Sydney, NSW 2007, Australia
}

\author{
S. Mahmoodian, R. C. McPhedran, and C. Martijn de Sterke \\ Centre for Ultrahigh bandwidth Devices for Optical Systems and School of Physics \\ University of Sydney, NSW 2006, Australia
}

(Dated: June 23, 2009)

\begin{abstract}
We consider the modal fields and resonance frequencies of composite defects in $2 \mathrm{D}$ photonic crystals (PCs). Using an asymptotic method based on Green's functions we show that the coupling matrices for the composite defect can be represented as circulant or block circulant matrices. Using the properties of these matrices, specifically that their eigenvectors are independent of the values of the matrix elements, we obtain modal properties such as dispersion relations, modal cutoff, degeneracy and symmetry of the mode fields. Using our formulation we investigate defects arranged on the vertices of regular polygons as well as PC ring resonators with defects arranged on the edges of polygons. Finally, we discuss the impact of band-edge degeneracies on composite-defect modes.
\end{abstract}

PACS numbers: 42.70.Qs, $42.25 . \mathrm{Bs}, 42.25 . \mathrm{Fx}$

\section{INTRODUCTION}

Many of the most interesting and important applications of photonic crystals (PCs) involve the use of defects in the lattice to create resonant cavities. These resonators are created by modifying the physical properties of the $\mathrm{PC}$ in a localised region, for example by changing the radius, position, or refractive index of the photonic crystal elements. A defect state created in this way is localised to the vicinity of the perturbation, and possesses a frequency that evolves from a band-edge into the photonic crystal band-gap as the strength of the perturbation increases. A natural extension of this idea is to modify the geometry of the PC, not at a single localised source, but at several, thereby creating a composite defect state. Composite defect states can form the basis of photonic crystal circuits; in particular they have important applications as add-drop filters [1-3] and cavities for laser sources $[4,5]$. These clusters can however be difficult to model: not only may the cluster consist of a great many interacting defects, but the desired state may exist close to the band-edge where the fields are markedly extended from the source of the perturbation.

Our goal here is to study such composite defect states as they evolve from the vicinity of a band-edge into a band gap. We analyse the structure of the fields as well as the distribution of mode frequencies of the composite state. In some situations many of the properties of the composite eigenstate associated with the cavity are well known: for example, for the case of two identical defects the eigenstates take the form of a symmetric/antisymmetric pair with frequencies equally spaced about the frequency of the individual defects provided that the

*Electronic address: Kokou.Dossou@uts.edu. au state is far from a band-edge. This observation is independent of the strength of the perturbation causing the defect, and of the spacing between the two defect sources. In this paper we generalise this result to clusters containing multiple defects, showing that this structure of the eigenstate is a necessary property of any rotationally symmetric cluster of identical defects. Moreover, the frequencies of the composite state can be accurately predicted using this model, even near the band-edge. We also examine the creation and evolution of the modes of composite states, predicting and explaining the cut-off of higher-order modes, as well as the properties (frequency and field structure) of fundamental modes.

Perturbation theory has been previously used to analyse the effect of small changes to dielectric interfaces [6] or photonic crystal waveguides [7]. Defect states created by isolated perturbations in infinitely extended photonic crystals were studied in Ref. [8]. Here we consider the properties of defect modes created by multiple perturbations in arbitrary extended 2D photonic crystals; this problem is intrinsically vectorial which leads to novel phenomena. We use an asymptotic treatment, based on the band-edge expansion of the Green function, to develop a semi-analytical formalism for describing the composite defect state. We then apply the rotational symmetry of the cluster to reduce the analysis to a single defect in a way that is very similar to the reduction of translationally periodic PCs to a unit cell. We find that for a nondegenerate band-edge, modelling a $\mathrm{PC}$ ring arranged as a regular polygon with $N_{e}$ edges and consisting of $N=N_{e} p$ defect cylinders (see Fig. 1), leads to a block circulant matrix problem for the composite eigenstate. The solution technique for this type of eigenproblem provides a powerful tool for the calculation of the eigenvectors because it reduces the original spectral problem of order $N_{e} p$ to $N_{e}$ decoupled eigenproblems of order $p$ [9-11]. We also generalize this method to eigenstates of defect 

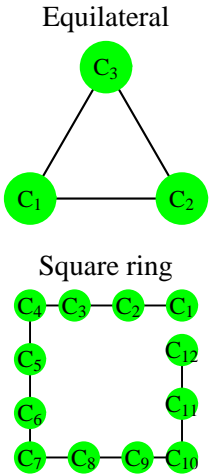

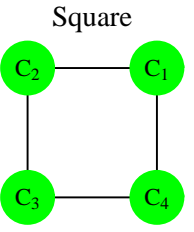

Square with centre

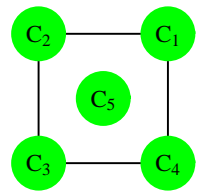

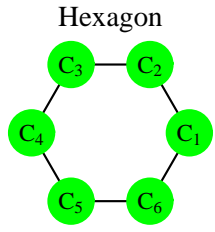

Rectangle

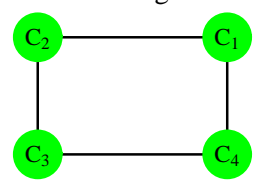

FIG. 1: (Color online) Symmetric structures considered here: For composite defects shaped as a regular polygon (equilateral, square, hexagon), the defect cylinders are in geometrically equivalent position. The square ring is also rotationally periodic and any three consecutive cylinders can be taken as a unit cell. The centre of the square centred structure is in a distinct geometric position. The rectangle is less symmetric.

modes arising from degenerate bands.

Throughout this paper we concentrate on modes created by weak perturbations. This approach allows us to elucidate the character of the modes near the band-edge - without such a treatment it would be difficult to say, for example, which modes approach cut-off and which approach the band-edge asymptotically. Although we focus on this "loose-binding" limit, the main results about the spectral properties of the defect modes are based only on the structure of the associated matrices (circulant, block circulant, real symmetric...) rather than the numerical values of their entries, which depend on coupling strength. Accordingly they can be generalized to other models such as such as the tight binding model [12] which leads to matrices with similar profiles. To validate our results, we compare the prediction of the asymptotic techniques to a full numerical calculation based on the FSS method [13-15].

The structure of the paper is as follows. In section II we describe the asymptotic behaviour of the Green function near a gap edge. We then use Green's theorem to formulate the problem for a defect near a nondegenerate band-edge in section III, presenting a detailed derivation for TM polarization and briefly outlining its generalization to TE polarization. The application of this theory to a double defect and symmetric clusters with an arbitrary number of defects follows in sections IV and V. In section VI we discuss the extension of this treatment to degenerate band-edges.

\section{ASYMPTOTIC EXPANSION OF THE GREEN FUNCTION NEAR A GAP EDGE}

We consider a $2 \mathrm{D}$ photonic crystal with a dielectric constant $\epsilon(\boldsymbol{r})$, consisting of a periodic array of circular cylinders with radius $a$ and separated by pitch $d$. We then perturb the properties of $N$ of these cylinders, modifying the overall dielectric constant to $\tilde{\epsilon}(\boldsymbol{r})$. Provided that the photonic crystal supports a complete band gap for some frequency range, this perturbation results in a composite defect state, with frequency $\omega$, that evolves from a bandedge into the gap.

The subsequent analysis relies on the Green function for the system near the edge of the band gap. The Green function may be expanded in terms of the eigenfunctions of the unperturbed system [8], which are the Bloch modes $\psi_{m}(\boldsymbol{k}, \boldsymbol{r})$, with $\boldsymbol{k}$ being the Bloch wavenumber:

$$
G\left(\boldsymbol{r}, \boldsymbol{r}^{\prime} ; \omega\right)=\frac{c^{2}}{4 \pi^{2}} \sum_{m} \int_{\mathrm{BZ}} \frac{\psi_{m}(\boldsymbol{k}, \boldsymbol{r}) \psi_{m}^{*}\left(\boldsymbol{k}, \boldsymbol{r}^{\prime}\right)}{\omega^{2}-\omega_{m}^{2}(\boldsymbol{k})} d^{2} \boldsymbol{k},
$$

where $c$ is the speed of light and the integration extends over the first Brillouin zone (BZ). We assume that the Bloch modes $\psi_{m}(\boldsymbol{k}, \boldsymbol{r})$ are normalized such that

$$
\frac{1}{A_{\mathrm{WSC}}} \int_{\mathrm{WSC}} s(\boldsymbol{r})\left|\psi_{m}(\boldsymbol{k}, \boldsymbol{r})\right|^{2} d^{2} \boldsymbol{r}=1,
$$

where $s(\boldsymbol{r})=\varepsilon(\boldsymbol{r})$ when $\psi(\boldsymbol{r})=E_{z}$ (TM or $E_{\|}$polarization) while $s(\boldsymbol{r})=1$ for $\psi(\boldsymbol{r})=H_{z}$ (TE or $H_{\|}$polarization) [8]. In the following analysis we derive expressions for the composite state in TM polarization; the TE case follows with only minor changes. The integration is evaluated over the Wigner-Seitz cell (WSC) and $A_{\mathrm{WSC}}$ is the area of WSC. We now denote the band on the edge of the gap by $m=L$, and consider the common situation in which a band has multiple extrema at $\boldsymbol{k}=\boldsymbol{k}_{L, l}$ for $l=1,2, \ldots, \nu_{L}$. These extrema are typically high symmetry points in the BZ. The band-edge frequency we then denote as $\omega_{L}$. The band-edge itself is assumed to be quadratic with principal curvatures $C_{L, x}$ and $C_{L, y}$ having a common sign; it is convenient to introduce the geometric mean curvature $C_{L}=\operatorname{sign}\left(C_{L, x}\right)\left(C_{L, x} C_{L, y}\right)^{1 / 2}$. The difference in frequency between the defect state and the band-edge is denoted by $\delta \omega=\omega-\omega_{L}$.

In the vicinity of the band-edge, the contribution from the band-edge dominates the sum in Eq. (1) [8]. We then have the leading order estimate for the Green function near the gap edge [8]

$$
\begin{aligned}
G\left(\boldsymbol{r}, \boldsymbol{r}^{\prime} ; \omega\right)= & -\frac{c^{2} C_{L} \theta_{L}}{2 \pi \omega_{L}} \sum_{l=1}^{\nu_{L}} K_{0}\left(b\left\|\boldsymbol{\sigma}_{\boldsymbol{k}_{L, l}}^{-1}\left(\boldsymbol{r}-\boldsymbol{r}^{\prime}\right)\right\|\right) \\
& \times \psi_{L}\left(\boldsymbol{k}_{L, l}, \boldsymbol{r}\right) \psi_{L}^{*}\left(\boldsymbol{k}_{L, l}, \boldsymbol{r}^{\prime}\right)+O\left((\delta \omega)^{0}\right),
\end{aligned}
$$

where $b=\sqrt{-2 C_{L} \delta \omega}$ - the negative sign under the square root reflects the physical observation that the change in frequency $\delta \omega$ must bring the defect state into the gap. The quantity $\theta_{L}$ is such that $2 \pi \theta_{L}$ is the interior angle of the BZ at each point $\boldsymbol{k}=\boldsymbol{k}_{L, l}$ and $\boldsymbol{\sigma}_{L, l}=\operatorname{diag}\left\{\left(C_{L, y} / C_{L, x}\right)^{1 / 4},\left(C_{L, x} / C_{L, y}\right)^{1 / 4}\right\}$ is a scaling matrix that takes into account the possibility of the band-edge having different principal curvatures. The leading order approximation (3) contains two important 


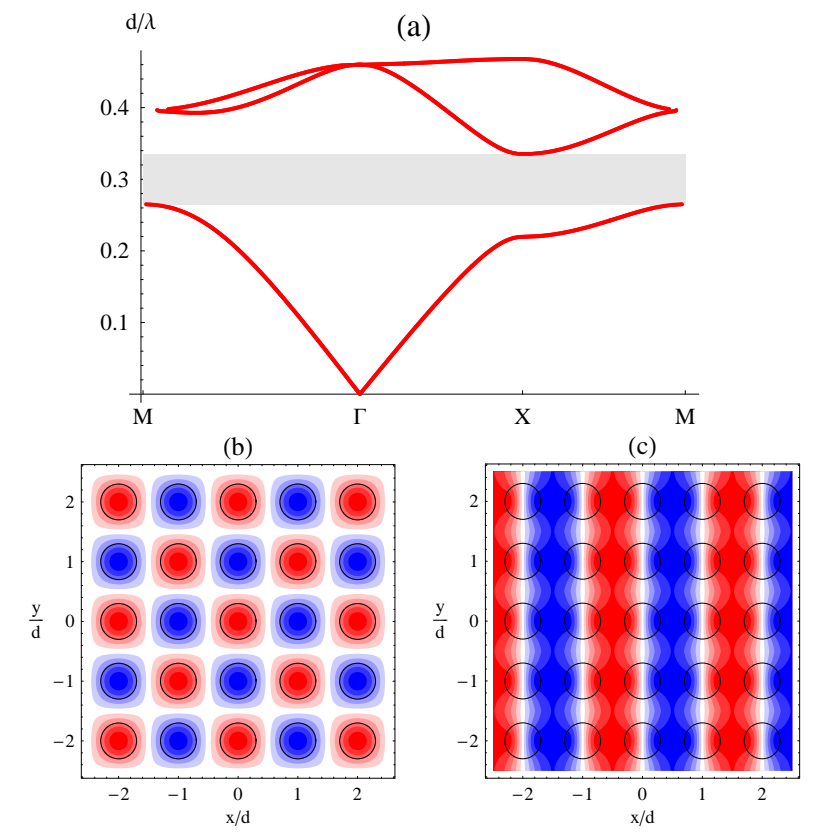

FIG. 2: (Color online) (a) Band diagram for a square array of cylinder rods of normalized radius $a / d=0.3$ and refractive index $n_{c}=3$ in a background of refractive index $n_{b}=1$. The $\mathrm{PC}$ is operated in TM polarization. (b) Bloch modes at the lower edge ( $M$ point) and (c) upper edge ( $X$ point) of the first band gap.

elements: an envelope function $\left(K_{0} b\left|\boldsymbol{\sigma}_{L, l}^{-1}\right| \boldsymbol{r}-\boldsymbol{r}^{\prime} \mid\right)$ that decays exponentially with increasing distance from the source point, and a combination of the band-edge Bloch $\operatorname{modes} \psi_{L}\left(\boldsymbol{k}_{L, l}, \boldsymbol{r}\right) \psi_{L}^{*}\left(\boldsymbol{k}_{L, l}, \boldsymbol{r}^{\prime}\right)$.

\section{FORMULATION FOR DEFECT NEAR A NONDEGENERATE BAND-EDGE}

There is only one linearly independent Bloch mode at a nondegenerate band-edge and this makes our perturbation theory relatively simple. Non-degeneracy at a bandedge can occur, for instance, at the lower edge of the first band gap for a square array of circular inclusions (see Fig. 2), and the composite defect state moves into the gap if the perturbation has the effect of lowering the refractive index contrast (this is sometimes known as negative defect) [8]. We consider the composite defect mode created by perturbations of the dielectric function in the vicinity of $N$ cylinders, labelled $C_{1}, C_{2}, \ldots, C_{N}$, and located at positions given by the vectors $\boldsymbol{o}_{1}, \boldsymbol{o}_{2}, \ldots, \boldsymbol{o}_{N}$.

The field $\psi(\boldsymbol{r})$ of the composite defect may be derived using Eq. (3) in Green's theorem, applying boundary conditions appropriate to the polarization under consideration. For TM polarization, the defect mode, $\psi(\boldsymbol{r})$, satisfies the Helmholtz equation

$$
\Delta \psi(\boldsymbol{r})+\frac{\omega^{2}}{c^{2}} \tilde{\varepsilon}(\boldsymbol{r}) \psi(\boldsymbol{r})=0 .
$$

By applying Green's theorem, the resulting expression for the defect field is

$$
\psi(\boldsymbol{r})=-\frac{\omega^{2}}{c^{2}} \sum_{j^{\prime}=1}^{N} \int_{C_{j^{\prime}}} \delta \varepsilon\left(\boldsymbol{r}^{\prime}\right) \psi\left(\boldsymbol{r}^{\prime}\right) G\left(\boldsymbol{r}, \boldsymbol{r}^{\prime} ; \omega\right) d^{2} \boldsymbol{r}^{\prime}
$$

with $\delta \varepsilon(\boldsymbol{r})=\tilde{\varepsilon}(\boldsymbol{r})-\varepsilon(\boldsymbol{r})$. Near each defect cylinder $C_{j}$ we now expand in terms of the basis of Bloch modes on the band-edge; for a nondegenerate band-edge, the basis is reduced to a single element $\psi_{L}\left(\boldsymbol{k}_{L}, \boldsymbol{r}\right)$. If we have a single defect cylinder we can use the leading order approximation [8] $\psi(\boldsymbol{r}) \approx \psi_{L}\left(\boldsymbol{k}_{L}, \boldsymbol{r}\right)$. However, in the case of multiple defect cylinders $C_{1}, C_{2}, \ldots, C_{N}$, we can write

$$
\psi(\boldsymbol{r}) \approx B_{j} \psi_{L}\left(\boldsymbol{k}_{L}, \boldsymbol{r}\right), \quad \boldsymbol{r} \in C_{j} .
$$

The estimate (6) is a leading-order expression for the fields for small perturbations, and so near the band-edge no new approximations have been made beyond what was necessary to obtain (3). Substituting (3) and (6) into (5), we obtain an equation for the unknown expansion coefficients $B_{j}$ :

$$
\begin{array}{r}
B_{j} \psi_{L}\left(\boldsymbol{k}_{L}, \boldsymbol{r}\right)=\frac{\omega_{L} C_{L}}{2 \pi} \sum_{j^{\prime}=1}^{N} B_{j^{\prime}} \psi_{L}\left(\boldsymbol{k}_{L}, \boldsymbol{r}\right) \\
\times \int_{C_{j^{\prime}}} \delta \varepsilon\left(\boldsymbol{r}^{\prime}\right) \psi_{L}\left(\boldsymbol{k}_{L}, \boldsymbol{r}^{\prime}\right) \psi_{L}^{*}\left(\boldsymbol{k}_{L}, \boldsymbol{r}^{\prime}\right) K_{0}\left(b\left\|\boldsymbol{r}-\boldsymbol{r}^{\prime}\right\|\right) d^{2} \boldsymbol{r}^{\prime}
\end{array}
$$

In deriving Eq. (7), we have made some simplifications which come from the nondegeneracy assumption: $\boldsymbol{\sigma}_{\boldsymbol{k}_{L, l}}$ is the identity matrix, $\psi_{L}\left(\boldsymbol{k}_{L, l}, \boldsymbol{r}\right)=\psi_{L}\left(\boldsymbol{k}_{L}, \boldsymbol{r}\right)$ and $\theta_{L} \nu_{L}=$ 1.

The problem can be significantly simplified by employing asymptotic approximations for the envelope function $K_{0}$ of the overlap integrals in (7). For two defect different cylinders $C_{j}$ and $C_{j^{\prime}}$, i.e., $j \neq j^{\prime}$, we make the approximation for $\boldsymbol{r} \in C_{j}$ and $\boldsymbol{r}^{\prime} \in C_{j^{\prime}}$

$$
K_{0}\left(b\left\|\boldsymbol{r}-\boldsymbol{r}^{\prime}\right\|\right) \approx K_{0}\left(b\left\|\boldsymbol{o}_{j}-\boldsymbol{o}_{j^{\prime}}\right\|\right) .
$$

Alternatively, for $j=j^{\prime}$ we must use the small-argument asymptotic expression

$$
K_{0}(z)=-\ln z+\ln \mathcal{A}
$$

where $\ln \mathcal{A}=O\left(z^{0}\right)$.

Substitution of these expressions into (7) and elimination of all higher-order terms leads to a first order relation for the unknown expansion coefficients $B_{j}$

$$
B_{j} \psi_{L}\left(\boldsymbol{k}_{L}, \boldsymbol{r}\right)=\frac{2}{s \mathcal{S}} \frac{\delta \mathcal{E}}{\mathcal{E}_{L}} \sum_{j^{\prime}=1}^{N} \beta_{j, j^{\prime}} B_{j^{\prime}} \psi_{L}\left(\boldsymbol{k}_{L}, \boldsymbol{r}\right),
$$

with

$$
\beta_{j, j^{\prime}}=\left\{\begin{array}{l}
-\frac{\ln |\delta \omega / \mathcal{A}|}{2} \text { if } j^{\prime}=j, \\
K_{0}\left(b\left\|\boldsymbol{o}_{j}-\boldsymbol{o}_{j^{\prime}}\right\|\right) \text { if } j^{\prime} \neq j .
\end{array}\right.
$$


In Eq. (10) the parameters $\mathcal{S}$ and $s$ are defined as

$$
\mathcal{S}=\frac{4 \pi}{\omega_{L}\left|C_{L}\right| A_{\mathrm{WSC}}} \text { and } s=\operatorname{sign} C_{L}
$$

$\mathcal{S}$ is referred to as the defect inertia [8]; the symbol $\mathcal{E}_{L}$ represents the electric energy of the Bloch mode within the Wigner-Seitz cell and $\delta \mathcal{E}$ is the perturbation to the electric energy caused by the defect:

$$
\begin{aligned}
\mathcal{E}_{L} & =\int_{\mathrm{WSC}} \varepsilon\left(\boldsymbol{r}^{\prime}\right)\left|\psi_{L}\left(\boldsymbol{k}_{L, 1}, \boldsymbol{r}^{\prime}\right)\right|^{2} d^{2} \boldsymbol{r}^{\prime}, \\
\delta \mathcal{E} & =\int_{C_{j^{\prime}}} \delta \varepsilon\left(\boldsymbol{r}^{\prime}\right)\left|\psi_{L}\left(\boldsymbol{k}_{L}, \boldsymbol{r}^{\prime}\right)\right|^{2} d^{2} \boldsymbol{r}^{\prime}
\end{aligned}
$$

Because of the quasi-periodicity of the Bloch mode, $\delta \mathcal{E}$ has the same value for all defect cylinders $C_{j^{\prime}}$. Relation (10) has the form of a coupling equation in the basis of Bloch modes.

By writing Eq. (10) at each defect site $j$, we obtain a matrix system

$$
(\boldsymbol{M}-\boldsymbol{I}) \boldsymbol{B}=\mathbf{0},
$$

where $\boldsymbol{I}$ and $\mathbf{0}$ are respectively the identity matrix and the zero vector of order $N ; \boldsymbol{B}$ is a vector of components, i.e., $\boldsymbol{B}=\left[B_{1}, \ldots, B_{N}\right]^{T}$, and $\boldsymbol{M}$ is a square matrix of order $N$ with coefficients defined as

$$
M_{j, j^{\prime}}=\frac{2}{s \mathcal{S}} \frac{\delta \mathcal{E}}{\mathcal{E}_{L}} \beta_{j, j^{\prime}}
$$

The matrix $\boldsymbol{M}$ is real and symmetric. The real eigenvalues $\xi_{s}$ of $\boldsymbol{M}$ depend on the frequency change $\delta \omega$ through the nonlinear relation (11). In general, the modes of Eq. (15), if they exist, are shifted from the band-edge by $\delta \omega$, according to the solutions of the defect dispersion equation

$$
\xi_{s}(\delta \omega)=1
$$

and the corresponding eigenvectors of $\boldsymbol{M}$ yield the form of the modes.

Equations (15)-(17), which have been derived for TM polarization, can be applied also to TE polarization [8]; the change in the electric energy $\delta \mathcal{E} / \mathcal{E}_{L}$ can be obtained from $H_{z}$ with Maxwell's equations.

\section{APPLICATION: DEFECT DISPERSION AND CUTOFF OF DOUBLE DEFECT NEAR A NONDEGENERATE BAND-EDGE}

We consider a square array of cylinder rods of normalized radius $a / d=0.3$ and refractive index $n_{c}=3$ in a background of refractive index $n_{b}=1$. The PC is operated in TM polarization and, as shown in Fig. 2, its first band gap corresponds to the normalized frequencies $d / \lambda \in[0.265263,0.334947]$. The upper edge of the first
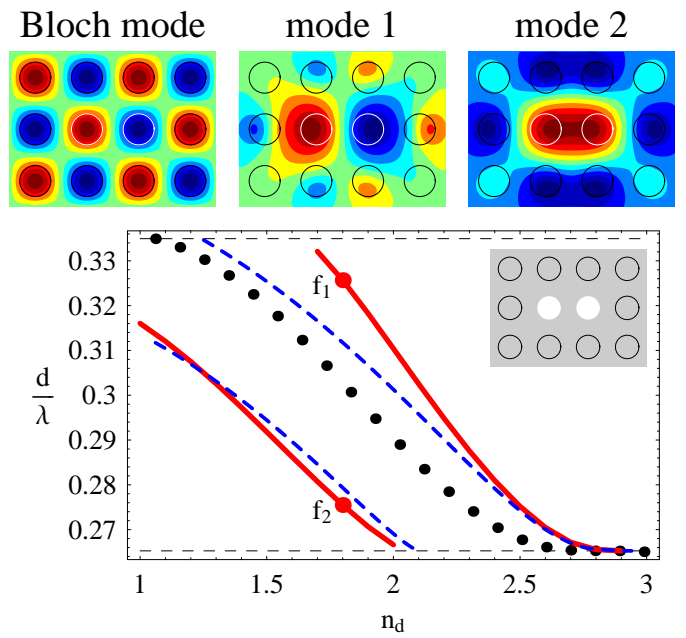

FIG. 3: (Color online) Evolution of a double defect: the separation between the defects is $d$. In the lower panel the continuous and dashed curves represent the defect dispersion obtained respectively with the FSS and the analytic methods. For a comparison, the dotted curve shows the dispersion (from FSS) for a single cylinder defect. The first plot in the top panel shows the Bloch mode at the lower gap edge while "mode 1" and "mode 2" are the defect modes associated, respectively, to the points $f_{1}$ and $f_{2}$ shown in the lower panel. mode 1
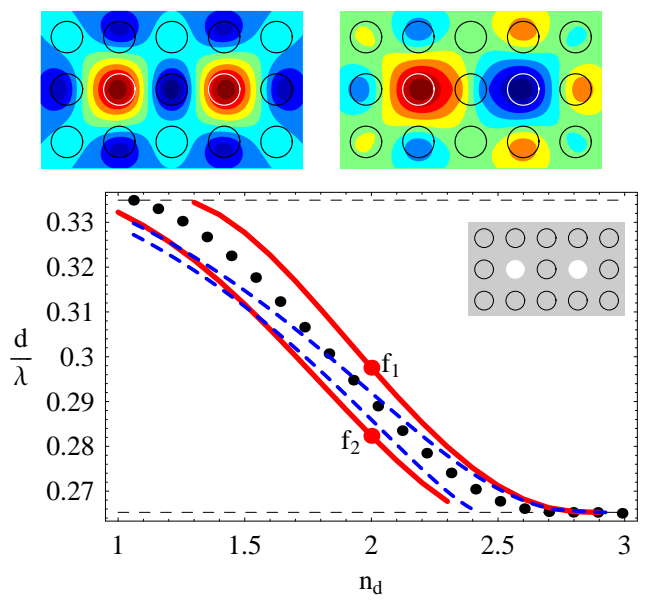

FIG. 4: (Color online) Evolution of a double defect: the separation between the defects is $2 d$.

band (lower edge of the first band gap) is nondegenerate while the lower edge of the second band (upper edge of the first band gap) is doubly degenerate.

To illustrate the application of the formalism we start with a simple example: that of a coupled pair of perturbations near a non-degenerate band-edge. We would expect that the composite state arises from a splitting of the solution of a single defect, and so consists of an even/odd symmetric pair. Here we have to find the nonzero solu- 

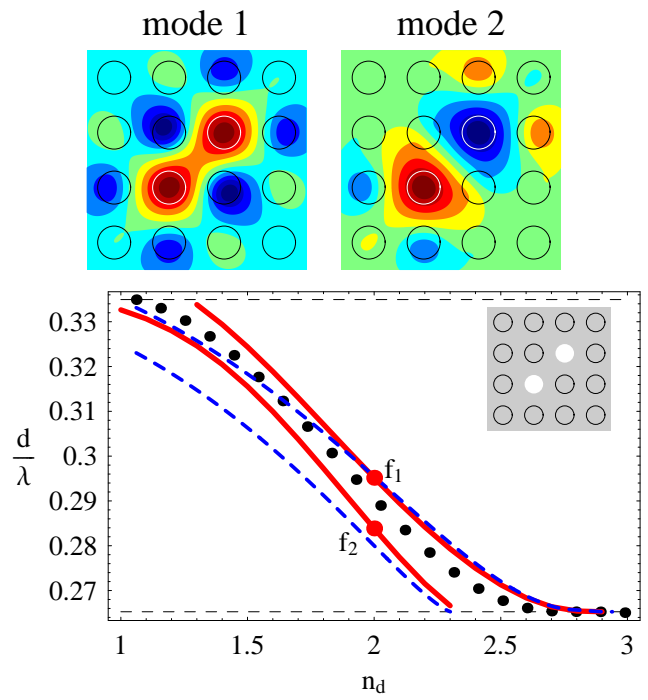

FIG. 5: (Color online) Evolution of a double defect: the separation between the defects is $d \sqrt{2}$ and the two defect are put along a diagonal direction of the square lattice.

tions to the homogeneous system from Eq. (15)

$$
\left[\begin{array}{cc}
\hat{M}_{0}-1 & \hat{M}_{1} \\
\hat{M}_{1} & \hat{M}_{0}-1
\end{array}\right]\left[\begin{array}{l}
B_{1} \\
B_{2}
\end{array}\right]=\left[\begin{array}{l}
0 \\
0
\end{array}\right]
$$

with $\hat{M}_{0}=M_{1,1}=M_{2,2}$ (identical defects) and $\hat{M}_{1}=$ $M_{1,2}=M_{2,1}$. This system has a non trivial solution only if $\hat{M}_{0}-1=-\hat{M}_{1}$ or $\hat{M}_{0}-1=\hat{M}_{1}$; these relations are respectively associated with the eigenvectors $\left[B_{1}, B_{2}\right]=$ $[1,1]$ and $\left[B_{1}, B_{2}\right]=[1,-1]$. In both cases, we need to solve a nonlinear equation to determine the frequency $\omega$. From the definition (16), the equation $\hat{M}_{0} \pm \hat{M}_{1}=1$ becomes

$$
\frac{s \mathcal{S}}{2} \frac{\mathcal{E}_{L}}{\delta \mathcal{E}}=-\frac{\ln |\delta \omega / \mathcal{A}|}{2} \pm K_{0}\left(\sqrt{-2 C_{L} \delta \omega}\left\|\boldsymbol{o}_{2}-\boldsymbol{o}_{1}\right\|\right)
$$

The parameter $\mathcal{A}$ can be evaluated by a fit to simulation data [8]. For a given defect energy $\delta \mathcal{E}$, we want to find the frequency $\delta \omega$ which satisfies the transcendental equation Eq. (19). Figures 3, 4 and 5 show the evolution of defects created by changing the refractive index of two cylinders from $n_{c}=3$ to $n_{d}<3$. For these examples the change in the electric energy $\mathcal{E}$ is negative and takes the form

$$
\delta \mathcal{E}=\left(n_{d}^{2}-n_{c}^{2}\right) \int_{C_{j^{\prime}}}\left|\psi_{L}\left(\boldsymbol{k}_{L}, \boldsymbol{r}^{\prime}\right)\right|^{2} d^{2} \boldsymbol{r}^{\prime}
$$

For a given defect refractive index $n_{d}$ the resonance frequencies are obtained using a full numerical calculation based on the FSS method [13-15] (continuous curves) and by solving Eq. (19) (dashed curves). For comparison, the dotted curve shows the defect evolution for a single cylinder defect (obtained with FSS). The horizontal dashed lines are the gap edges. The field plots in the top panel are obtained using the FSS method. For weak perturbations, i.e., the value of $n_{d}$ is near $n_{c}=3$, there is a good agreement between the full numerical calculation and our perturbation theory. For the defect dispersion curves presented in this paper, unless other notations are indicated in the figure caption, we will use continuous curves and dashed curves to represent the FSS and asymptotic results respectively.

There are two branches of solutions for the defect dispersion curves in Figs 3, 4 and 5. The upper branch corresponds to the problem $\hat{M}_{0}+\hat{M}_{1}=1$ and does not cutoff as $\delta \mathcal{E}$ approaches zero, i.e., as $n_{d}$ tends to $n_{c}=3$; we refer to the solutions on this branch as fundamental modes. Since the corresponding eigenvector is $\left[B_{1}, B_{2}\right]=[1,1]$, the fundamental mode has even symmetry when the Bloch mode fields at the two defect cylinders have the same phase (see Figs 4 and 5) while it has odd symmetry when the fields have opposite phases (see Fig. 3). This behaviour is consistent with that for coupled photonic waveguide, where the topology of the fundamental mode depends on the spacing between the guides [16].

In contrast to the fundamental mode, the lower branch, which consists of solutions of $\hat{M}_{0}-\hat{M}_{1}=1$ or $\left[B_{1}, B_{2}\right]=$ $[1,-1]$ (higher order modes), cuts off as $\delta \mathcal{E}$ tends to zero. These properties of the two branches can be explained with our perturbation theory. Near the gap edge the variable $\delta \omega$ has small values and when $\sqrt{\delta \omega}\left\|\boldsymbol{o}_{2}-\boldsymbol{o}_{1}\right\|$ is small enough, from the asymptotic expansion $K_{0}(z)=$ $-\ln z+\ln 2-\gamma+O\left(z^{2}\right)$, (where $\gamma$ is the Euler constant $\gamma=0.57721566 \ldots)$ we have

$$
K_{0}\left(\sqrt{-2 C_{L} \delta \omega}\left\|\boldsymbol{o}_{2}-\boldsymbol{o}_{1}\right\|\right)=-\frac{\ln |\delta \omega Q|}{2}+O\left(|\delta \omega|^{2}\right)(21)
$$

with

$$
Q=e^{2 \gamma}\left|C_{L}\right|\left\|\boldsymbol{o}_{2}-\boldsymbol{o}_{1}\right\|^{2} / 2 .
$$

It follows from Eq. (21) and the definitions (11) and (16) that, near the gap edge, the logarithmic singularity $2 \delta \mathcal{E} \ln |\delta \omega| /\left(2 s \mathcal{S} \mathcal{E}_{L}\right)$ is the dominant term of $\hat{M}_{0}$ and $\hat{M}_{1}$. However with the equations $\hat{M}_{0}-\hat{M}_{1}=1$, these two logarithmic singularities cancel each other while they add constructively with $\hat{M}_{0}+\hat{M}_{1}=1$. We now examine each case separately.

If $\hat{M}_{0}-\hat{M}_{1}=1$, with the cancellation of the logarithmic singularity, $\hat{M}_{0}(\delta \omega)-\hat{M}_{1}(\delta \omega)$ becomes a product of $\delta \mathcal{E}$ by a function which is regular with respect to $\delta \omega$ and thus bounded for small values of $|\delta \omega|$, i.e., near the gap edge. As a consequence, there exists a minimum defect strength $\delta \mathcal{E}_{\text {cutoff }}$ such that the equation $\hat{M}_{0}(\delta \omega)-\hat{M}_{1}(\delta \omega)=1$ has no solution near the gap edge if $|\delta \mathcal{E}|<\left|\delta \mathcal{E}_{\text {cutoff }}\right|$. By substituting the leading term of Eq. (21) into Eq. (19), we may estimate the minimum defect strength $\delta \mathcal{E}_{\text {cutoff }}$ for the creation of the high order mode

$$
\frac{\delta \mathcal{E}_{\text {cutoff }}}{\mathcal{E}_{L}}=\frac{s \mathcal{S}}{\ln (Q \mathcal{A})}
$$


Figure 6 shows a good agreement between the FSS calculations and the cutoff energy change given by Eq. (23). Since, from Eq. (22), $Q$ is proportional to the square of the defect separation $\left\|\boldsymbol{o}_{2}-\boldsymbol{o}_{1}\right\|$, the higher order mode becomes progressively harder to create as the two defects get closer.

However for a large value of $\left\|\boldsymbol{o}_{2}-\boldsymbol{o}_{1}\right\|$, the small argument asymptotic (21) is valid only for very small values of $|\delta \omega|$. The large argument approximation $K_{0}(z) \approx$ $\sqrt{\pi} \exp (-z) / \sqrt{2 z}$ shows that $\hat{M}_{1}$ becomes negligible with respect to $\hat{M}_{0}$ and the solutions of the equation $\hat{M}_{0} \pm$ $\hat{M}_{1}=1$ approaches the solutions of the equation $\hat{M}_{0}=1$ for a single defect cylinder when $|\delta \omega|\left\|\boldsymbol{o}_{2}-\boldsymbol{o}_{1}\right\|$ is large enough. Thus the higher order mode becomes easier to create as the distance between the defect increases.

If $\hat{M}_{0}+\hat{M}_{1}=1$, we can always find a solution to the equation near the gap edge as $\delta \mathcal{E}$ tends to zero because of the dominant logarithmic term $\ln |\delta \omega|$. For the fundamental mode, the asymptotic relation Eq. (21) then leads to the dispersion relation

$$
|\delta \omega|=\frac{\sqrt{\mathcal{A}}}{\sqrt{Q}} \exp \left(-\frac{s \mathcal{S} \mathcal{E}_{L}}{2 \delta \mathcal{E}}\right)
$$

We find then that the frequency shift $\delta \omega$ follows an exponential law. The argument of the exponential function is half that of a single defect [8] and this is due to the fact that the presence of two defect cylinders doubles the perturbation of the energy in the system leading to a factor of 2 in the denominator of exponential argument in Eq. (24). From Eq. (22), the prefactor $\sqrt{\mathcal{A}} / \sqrt{Q}$ in Eq. (24) is inversely proportional to the separation $\left\|\boldsymbol{o}_{2}-\boldsymbol{o}_{1}\right\|$ between the defects. Figures 3 and 4 show that the frequency of the fundamental mode corresponding to the defect separation $d$ shifts into the gap significantly faster than for the double defect with separation $2 d$; this is also confirmed by Fig. 7 which shows that the ratio between the frequency shifts converges to 2 as $n_{d}$ tends to $n_{c}=3$.

\section{ROTATIONALLY SYMMETRIC COMPOUND DEFECTS AND CIRCULANT MATRICES}

\section{A. Polygonal composite defects}

We have seen that for a double defect, the eigenvector $\boldsymbol{B}=\left[B_{1}, B_{2}\right]$ does not depend on the coupling coefficients, i.e., the numerical values of the matrix $\boldsymbol{M}$. This is a well-known property of structures consisting of two identical resonators and it provides the basis for the classification of the defect mode symmetry as even or odd. We now show that this structural stability, i.e., the fact that eigenvectors $\boldsymbol{B}$ are independent of the values of the coupling coefficients of the matrix $\boldsymbol{M}$ can be generalised to multiple defects. We consider rotationally symmetric composite defects in the form of a regular polygon such

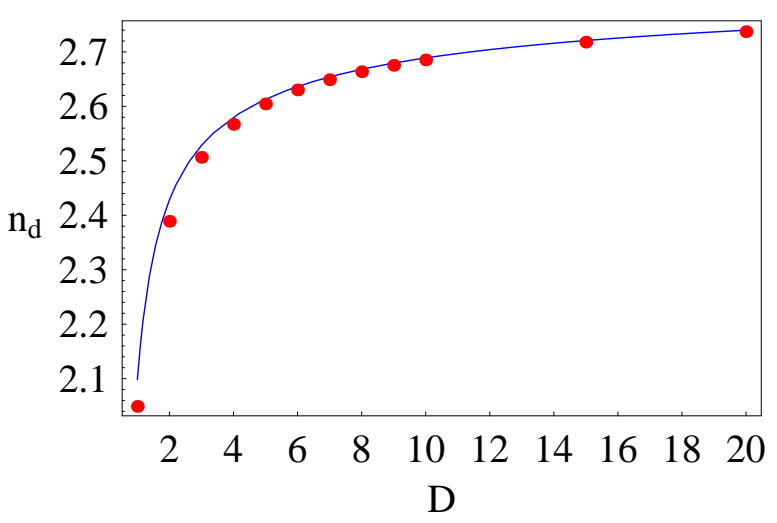

FIG. 6: (Color online) Cutoff value for the higher order mode of a double defect versus defect separation $D=\left\|\boldsymbol{o}_{2}-\boldsymbol{o}_{1}\right\|$. The dots represent the FSS results while the continuous curve is the solutions given by Eqs (20) and (23).

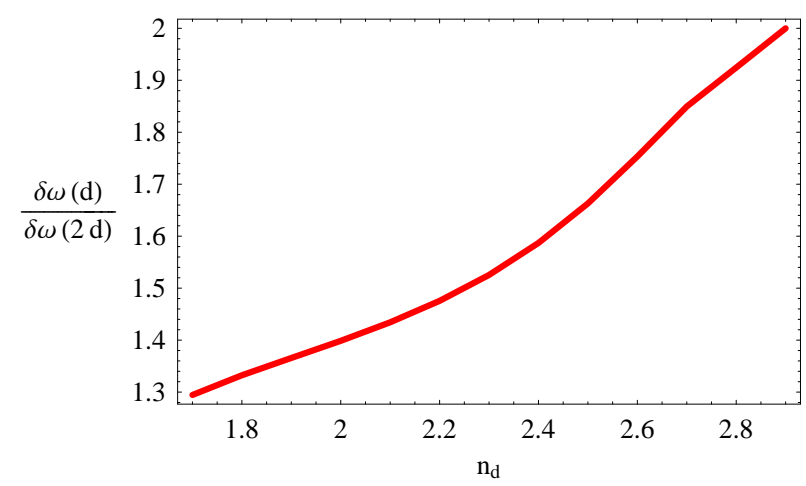

FIG. 7: (Color online) Ratio of the frequency shifts $\delta \omega(d)$ and $\delta \omega(2 d)$ created by two defects of refractive index $n_{d}$ and separated by distances $d$ and $2 d$ respectively (see Figs 3 and 4 ). For a weak defect, i.e., $n_{d} \approx n_{c}=3$, the ratio is close to 2 .

as the equilateral triangle, the square and the hexagon shown in Fig. 1. In these cases all the defect cylinders are in geometrically equivalent positions so that the matrix $\boldsymbol{M}$ takes a particular form, that of a circulant matrix,

$$
\boldsymbol{M}=\left[\begin{array}{ccccc}
\hat{M}_{0} & \hat{M}_{1} & \hat{M}_{2} & \cdots & \hat{M}_{N-1} \\
\hat{M}_{N-1} & \hat{M}_{0} & \hat{M}_{1} & \cdots & \hat{M}_{N-2} \\
\vdots & \vdots & \vdots & \vdots & \vdots \\
\hat{M}_{1} & \cdots & \hat{M}_{N-2} & \hat{M}_{N-1} & \hat{M}_{0}
\end{array}\right]
$$

with $\hat{M}_{j-1}=M_{1, j}$ for $j=1, \ldots, N$. A circulant matrix, a close relative of the Toeplitz matrix, is a square matrix with each row cyclically shifted one element to the right with respect to the preceding row. The eigenvectors of a circulant matrix of order $N$ are (see Ref. [10])

$$
\boldsymbol{B}_{j}=\left[1, \rho_{j}, \rho_{j}^{2}, \ldots, \rho_{j}^{N-1}\right]^{T},
$$


where, for $j=1, \ldots, N, \rho_{j}$ is a $N^{\text {th }}$ root of unity: $\rho_{j}=$ $e^{2 \pi i(j-1) / N}$.

These eigenvectors are independent of the values of the matrix elements. This means that the shape stability property exhibited by a nondegenerate two-defect system generalizes to compound defects with a rotationally symmetric polygonal configuration. We note that the shape of the composite defect needs to be consistent with the lattice, for example no hexagonal defect shape is possible in a square lattice.

The eigenvalues $\xi_{j}$ are real because the matrix $\boldsymbol{M}$ is real and symmetric. Since $\rho_{N+1-j}=\rho_{j}^{*}$ and $\boldsymbol{B}_{N+1-j}=$ $\boldsymbol{B}_{j}^{*}$, it follows that $\xi_{N+1-j}=\xi_{j}$. This means that when $\rho_{j} \neq \rho_{N+1-j}$ (conjugate pair of roots), the eigenvalue $\xi_{j}=\xi_{N+1-j}$ corresponds to a doubly-degenerate mode of $\boldsymbol{M}$. A real root $\rho_{j}$ generally gives a non degenerate mode.

For each eigenvector $\boldsymbol{B}_{j}$, the associated eigenvalue can be written as $\xi_{j}=\left[\hat{M}_{0}, \hat{M}_{1}, \cdots, \hat{M}_{N-1}\right]^{T} \cdot \boldsymbol{B}_{j}$, i.e., $\xi_{j}$ is the scalar product of $\boldsymbol{B}_{j}$ by the first row of the matrix $\boldsymbol{B}$. Note that the components of the first eigenvector $\boldsymbol{B}_{1}$ (fundamental mode) are equal, while the sum of the components is zero for the remaining eigenvectors $\boldsymbol{B}_{j}$, $j \in\{2, \ldots, N\}$ (high order modes).

As for the double defect, the coupling coefficients $\hat{M}_{0}, \hat{M}_{1}, \cdots, \hat{M}_{N-1}$ develop a logarithmic singularity near the gap edge, and the singularities add constructively only for the eigenvalue $\xi_{1}$ associated with the fundamental mode. As a consequence, the fundamental mode does not cutoff but follows an exponential law with an exponent $N$ times smaller than that of a single defect. The field profile of the fundamental mode has the same symmetry, with respect to the defect structure, as the Bloch mode. The higher order modes cutoff when $\delta \mathcal{E}$ tends to zero and, as for the double defect Eq. (19), there is no solution to the dispersion Eq. (17) if $|\delta \mathcal{E}|$ is below a specific threshold.

To illustrate this, we show examples of a quadruple $2 \times$ 2 defect (see Figs 8 and 9 ) in a square array. The solution to the eigenvalue problem yields two degenerate and two nondegenerate states, of which all but the fundamental mode are cut off for small defect strengths.

This type of analysis can be easily adapted to more general symmetric defect structures. For example, with a compound defect located on the vertices as well as at the centre of a rotationally symmetric polygon, (see Fig. 1 for an example of a centred square). The eigenvalue problem corresponding to the $N+1$ defect cylinders is

$$
\left[\begin{array}{cccc|c}
\hat{M}_{0} & \hat{M}_{1} & \cdots & \hat{M}_{N-1} & \hat{M}_{N} \\
\hat{M}_{N-1} & \hat{M}_{0} & \cdots & \hat{M}_{N-2} & \hat{M}_{N} \\
\vdots & \vdots & \vdots & \vdots & \vdots \\
\hat{M}_{1} & \hat{M}_{2} & \cdots & \hat{M}_{0} & \hat{M}_{N} \\
-- & -- & --- & -- \\
\hat{M}_{N} & \hat{M}_{N} & \cdots & \hat{M}_{N} & \hat{M}_{0}
\end{array}\right]\left[\begin{array}{c}
B_{1} \\
B_{2} \\
\vdots \\
B_{N} \\
--- \\
B_{N+1}
\end{array}\right]=\left[\begin{array}{c}
B_{1} \\
B_{2} \\
\vdots \\
B_{N} \\
--- \\
B_{N+1}
\end{array}\right]
$$

The matrix of Eq. (27) is obtained by appending a column and a row to a circulant matrix of order $N$; all

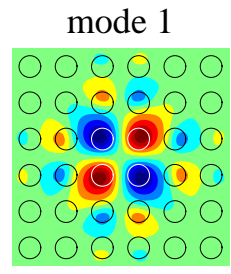

mode $2(\mathrm{~b})$
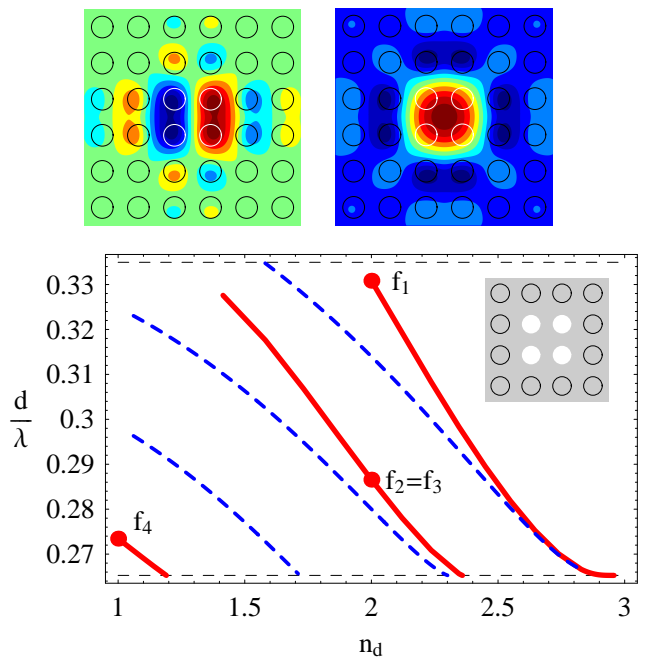

FIG. 8: (Color online) Evolution of a quadruple defect: the vertical and horizontal separations between the defect are $d$. We use the notations (a) and (b) to refer to a pair of degenerate modes.

the off-diagonal elements in the appended column and row are equal to each other. Since the sum of entries of any circulant matrix eigenvector in Eq. (26), except the fundamental $\boldsymbol{B}_{1}=[1,1, \ldots, 1]$, is zero, we obtain an eigenvector $\hat{\boldsymbol{B}}_{j}$ of problem (27) by appending zero to the eigenvectors $\boldsymbol{B}_{j}$, for $j=2, \ldots, N$, of Eq. (26). We find that

$$
\hat{\boldsymbol{B}}_{j}=\left[\begin{array}{c}
\boldsymbol{B}_{j} \\
0
\end{array}\right], \quad \text { for } j=2, \ldots, N
$$

yields $N-1$ eigenvectors for the eigenproblem Eq. (27) of order $N+1$ so we need to find only two more eigenvectors.

By noticing that the vectors $\left[\boldsymbol{B}_{1}^{T}, 0\right]^{T}$ and $[0, \ldots, 0,1]^{T}$ are not linear combinations of the modes $\hat{\boldsymbol{B}}_{j}$ in Eq. (28), we search for eigenvectors which are linear combinations of these new vectors

$$
\hat{\boldsymbol{B}}=a\left[\begin{array}{c}
\boldsymbol{B}_{1} \\
0
\end{array}\right]+b\left[\begin{array}{c}
0 \\
0 \\
\vdots \\
0 \\
1
\end{array}\right]=\left[\begin{array}{c}
a \\
a \\
\vdots \\
a \\
b
\end{array}\right] .
$$

It can be verified that $\hat{\boldsymbol{B}}$ is an eigenvector of Eq. (27) if and only if $[a, b]^{T}$ is a solution to the following eigen- 


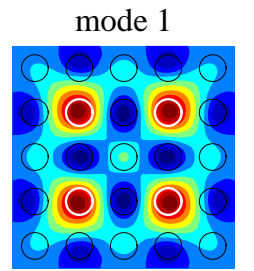

mode 2 (a)
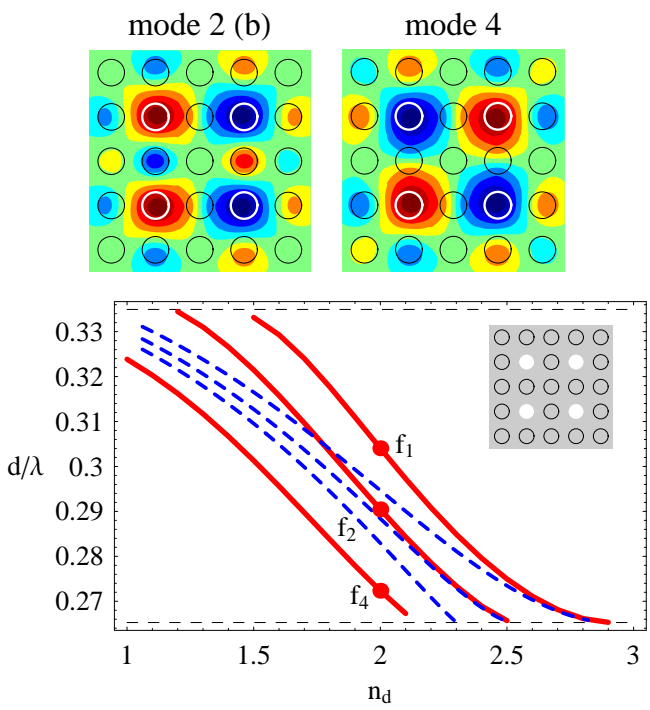

FIG. 9: (Color online) Evolution of a quadruple defect: the vertical and horizontal separations between the defect are $2 d$.

problem

$$
\left[\begin{array}{cc}
\sum_{j=0}^{N-1} \hat{M}_{j} & \hat{M} \\
N \hat{M} & \hat{M}_{0}
\end{array}\right]\left[\begin{array}{l}
a \\
b
\end{array}\right]=\xi\left[\begin{array}{l}
a \\
b
\end{array}\right]
$$

from which we deduce the remaining two eigenvectors.

As an example, we add a defect cylinder at the centre of the square defect cluster presented in Fig. 9. Figure 10 shows the defect dispersion of the resulting square centred defect structure. For comparison, the defect evolution for a square without a centre (see Fig. 9) is also shown (dashed curves) indicating that, as expected, the higher order modes created by the non-centred square structure are only weakly perturbed when a centre defect cylinder is added.

The profiles of the modes created by the centred square defect system are also plotted in Fig. 10. The panel of Fig. 10 labelled "mode 4" shows the new higher order mode; this mode is highly concentrated near the central defect cylinder $C_{N+1}$ indicating that it corresponds to a solution $(a, b)$ of Eq. (30) such that $a \approx 0$ which means that $(a, b)$ can be taken as $(a, b) \approx(0,1)$. The energy of the fundamental mode, labelled "mode 1 ", is nearly equally distributed between the defect sites and its profile shows that it is associated with a solution $(a, b)$ which satisfies $(a, b) \approx(1,1)$. These observations can be explained by the fact that the matrix of Eq. (30) is close to being a lower triangular matrix, i.e., the off-diagonal entry $M$ is smaller than the others, and the remaining entries satisfy $\sum_{j=0}^{N-1} \hat{M}_{j} \approx \hat{M}_{0}+N \hat{M}$ (the contribution of $\hat{M}_{0}$ is dominant on both sides). Since the eigenvectors of a lower triangular matrix of order 2 are $(0,1)$ and $(1,1)$ when the sums of elements in each row are equal, we can then expect the eigenvectors of Eq. (30) to satisfy approximately these properties.

The shape stability property of regular polygon arrangement of defects can also be extended to the rectangular profile (see Fig. 1) even though is matrix is not strictly "circulant". The $\boldsymbol{M}$-matrix for the rectangle shown in Fig. 1, has the form of a block circulant matrix:

$$
\boldsymbol{M}=\left[\begin{array}{cc:cc}
\hat{M}_{0} & \hat{M}_{1} & \hat{M}_{2} & \hat{M}_{3} \\
\hat{M}_{1} & \hat{M}_{0} & \hat{M}_{3} & \hat{M}_{2} \\
\hdashline--- & --- \\
\hat{M}_{2} & \hat{M}_{3} & \hat{M}_{0} & \hat{M}_{1} \\
\hat{M}_{3} & \hat{M}_{2} & \hat{M}_{1} & \hat{M}_{0}
\end{array}\right]=\left[\begin{array}{ll}
\boldsymbol{A}_{0} & \boldsymbol{A}_{1} \\
\boldsymbol{A}_{1} & \boldsymbol{A}_{0}
\end{array}\right]
$$

with $\hat{M}_{0}=M^{(1,1)}{ }_{11}, \hat{M}_{1}=M^{(1,2)}{ }_{11}, \hat{M}_{2}=M^{(1,3)}{ }_{11}$, $\hat{M}_{3}=M^{(1,4)} 11$. This matrix itself is not circulant, but instead consists of a circulant matrix of order 2 where the coefficients are matrices. For the matrix (31) we have the eigenproblem

$$
\left[\begin{array}{cc}
\boldsymbol{A}_{0}-\xi \boldsymbol{I} & \boldsymbol{A}_{1} \\
\boldsymbol{A}_{1} & \boldsymbol{A}_{0}-\xi \boldsymbol{I}
\end{array}\right]\left[\begin{array}{l}
\boldsymbol{w}_{1} \\
\boldsymbol{w}_{2}
\end{array}\right]=\left[\begin{array}{l}
\mathbf{0} \\
\mathbf{0}
\end{array}\right] .
$$

It is clear that

$$
\left[\begin{array}{l}
\boldsymbol{w} \\
\boldsymbol{w}
\end{array}\right] \text { and }\left[\begin{array}{c}
\boldsymbol{w} \\
-\boldsymbol{w}
\end{array}\right]
$$

are both eigensolutions provided that, for the former partitioned vector $\left(\left[\boldsymbol{w}^{T}, \boldsymbol{w}^{T}\right]^{T}\right)$, the vector $\boldsymbol{w}$ is an eigenvector of

$$
\boldsymbol{T}=\boldsymbol{A}_{0}+\boldsymbol{A}_{1}
$$

while for the latter partitioned vector $\left(\left[\boldsymbol{w}^{T},-\boldsymbol{w}^{T}\right]^{T}\right), \boldsymbol{w}$ is eigenvector of

$$
\boldsymbol{T}=\boldsymbol{A}_{0}-\boldsymbol{A}_{1}
$$

Since both $\boldsymbol{A}_{0}$ and $\boldsymbol{A}_{1}$ are circulant matrices of order 2, then according to Eq. (26), the vectors $\boldsymbol{w}_{1}=[1,1]^{T}$ and $\boldsymbol{w}_{2}=[1,-1]^{T}$ are eigenvectors of the matrices $\boldsymbol{A}_{0} \pm \boldsymbol{A}_{1}$. So the eigenvectors of $\boldsymbol{M}=\left[\begin{array}{ll}\boldsymbol{A}_{0} & \boldsymbol{A}_{1} \\ \boldsymbol{A}_{1} & \boldsymbol{A}_{0}\end{array}\right]$ are

$$
\left[\begin{array}{l}
\boldsymbol{w}_{j} \\
\boldsymbol{w}_{j}
\end{array}\right] \text { and }\left[\begin{array}{r}
\boldsymbol{w}_{j} \\
-\boldsymbol{w}_{j}
\end{array}\right], \quad \text { for } j=1,2
$$

i.e., the eigenvectors are $\boldsymbol{B}_{1}=[1,1,1,1]^{T}, \quad \boldsymbol{B}_{2}=$ $[1,-1,1,-1]^{T}, \quad \boldsymbol{B}_{3}=[1,1,-1,-1]^{T}$ and $\boldsymbol{B}_{4}=$ $[1,-1,-1,1]^{T}$. Thus the eigensystem of the matrix $\boldsymbol{M}$ of order 4 has been calculated by solving two reduced eigenproblems order 2 . 


\section{B. Photonic crystal rings}

In section $\mathrm{V}$ A the submatrices are of order 2 because the coupling terms around the rectangle repeat for every second defect. The underlying circulant matrix is also of order 2, however the analysis of symmetric composites can lead to more complicated block matrices which are based on circulant matrices and submatrices of arbitrary orders. For instance, in many applications, photonic crystal ring resonators [1-3] have a shape of a regular polygon with $N_{e}$ edges where, in addition to defect cylinders on the vertices, $p-1$ defect cylinders are placed on each edge so that there are $N=N_{e} \times p$ defect cylinders. Figure 1 illustrates a ring resonator with $N_{e}=4$ and $p=3$. For a nondegenerate band-edge, the analysis of such structures gives a matrix $M$ of the form

$$
\boldsymbol{M}=\left[\begin{array}{cccc}
\hat{\boldsymbol{M}}_{0} & \hat{\boldsymbol{M}}_{1} & \ldots & \hat{\boldsymbol{M}}_{N_{e}-1} \\
\hat{\boldsymbol{M}}_{N_{e}-1} & \hat{\boldsymbol{M}}_{0} & \ldots & \hat{\boldsymbol{M}}_{N_{e}-2} \\
\vdots & \vdots & \vdots & \vdots \\
\hat{\boldsymbol{M}}_{1} & \cdots & \hat{\boldsymbol{M}}_{N_{e}-1} & \hat{\boldsymbol{M}}_{0}
\end{array}\right]
$$

which is block circulant with $N_{e}$ columns containing the square submatrices $\hat{M}_{l}$ of order $p$. This particular form of the matrix $M$ reflects the fact that the geometry of the symmetric ring structure repeats itself with a periodicity of $p$. The procedures used to compute the eigenvectors (36) of the block circulant matrix $\boldsymbol{M}$ in Eq. (31) by solving the reduced size eigenproblems (34) and (35) can be generalized to block circulant matrices of arbitrary orders $[9,10]$ : for each root of unity $\rho_{j}=e^{2 \pi i(j-1) / N_{e}}$, with $j=1, \ldots, N_{e}$, let $\boldsymbol{T}_{j}$ be the matrix

$$
\boldsymbol{T}_{j}=\sum_{l=0}^{N_{e}-1} \rho_{j}^{l} \hat{\boldsymbol{M}}_{l}=\sum_{l=0}^{N_{e}-1} e^{2 \pi i l(j-1) / N} \hat{\boldsymbol{M}}_{l}
$$

Then for any eigenvalue $\xi$ of $\boldsymbol{T}_{j}$ with an associated eigenvector $\boldsymbol{w}$, is also an eigenvalue of the block matrix $\boldsymbol{M}$ and the corresponding eigenvector of $M$ is

$$
\boldsymbol{B}=\left[\begin{array}{c}
\boldsymbol{w} \\
\rho_{j} \boldsymbol{w} \\
\vdots \\
\rho_{j}^{N_{e}-1} \boldsymbol{w}
\end{array}\right]
$$

We now show that the matrices $\boldsymbol{T}_{j}$ are real symmetric or complex Hermitian. By using the fact that the matrix $\boldsymbol{M}$ is real symmetric and block circulant, together with the relation $\rho_{j}^{N_{e}-l}=\left(\rho_{j}^{l}\right)^{*}$ (derived from $\rho_{j}=e^{2 \pi i(j-1) / N_{e}}$ ) and the convention $\hat{\boldsymbol{M}}_{N_{e}}=\hat{\boldsymbol{M}}_{0}$, we can derive the following relations between the submatrices $\hat{M}_{l}$

$$
\begin{array}{ll}
\hat{\boldsymbol{M}}_{N_{e}-l} & =\hat{\boldsymbol{M}}_{l}^{T}, \text { for } l=0, \ldots, N_{e}-1 \\
\rho_{j}^{N_{e}-l} \hat{\boldsymbol{M}}_{N_{e}-l} & =\left(\rho_{j}^{l} \hat{\boldsymbol{M}}_{l}\right)^{H}
\end{array}
$$

where $T$ and $H$ denote respectively the transpose and Hermitian (conjugate transpose) operators.

We then have

$$
\begin{aligned}
\boldsymbol{T}_{j} & =\frac{1}{2} \sum_{l=0}^{N_{e}-1}\left(\rho_{j}^{l} \hat{\boldsymbol{M}}_{l}+\rho_{j}^{N_{e}-l} \hat{\boldsymbol{M}}_{N_{e}-l}\right) \\
& =\frac{1}{2} \sum_{l=0}^{N_{e}-1}\left(\rho_{j}^{l} \hat{\boldsymbol{M}}_{l}+\left(\rho_{j}^{l} \hat{\boldsymbol{M}}_{l}\right)^{H}\right) .
\end{aligned}
$$

which shows that $\boldsymbol{T}_{j}$ is real symmetric or complex Hermitian.

Thus each matrix $\boldsymbol{T}_{j}$ has $p$ linearly independent eigenvectors; the relation (39) will then give a complete set of $N_{e} \times p$ eigenvectors of $\boldsymbol{M}$ [10]. In general each $\boldsymbol{T}_{j}$ has $p$ distinct real eigenvalues. However for a conjugate pair of distinct roots $\rho_{j}$ and $\rho_{N_{e}+1-j}$, the Hermitian matrices $\boldsymbol{T}_{j}$ and $\boldsymbol{T}_{N_{e}+1-j}$ are also conjugate to each other, so that they have the same real eigenvalues which, as eigenvalues of the block matrix $\boldsymbol{M}$, can be associated with a pair of conjugate eigenvectors obtained from the corresponding modes of $\boldsymbol{T}_{j}$ and $\boldsymbol{T}_{N_{e}+1-j}$ through Eq. (39). For example, when $N_{e}=4$, we have $\rho_{j} \in\left\{ \pm 1, e^{ \pm \pi i / 2}\right\}$ and the two real roots generically give $p$ distinct eigenvalues each, while the conjugate pair will also provide $p$ doubly degenerate eigenvalues; thus in total we have $3 p$ eigenvalues for $\boldsymbol{M}$. Indeed, for the PC ring of Fig. 11, where $N_{e}=4$ and $p=3$, we have $3 p=9$ defect dispersion curves.

There are some similarities between a rotationally symmetric PC ring and a standard periodic PC. In our model, any $p$ consecutive defect cylinders can be seen as a unit cell and the whole ring can be generated by successive rotations of this cell by an angle $2 \pi / N_{e}$. The eigenvector (39) is then analogous to a Bloch mode of the rotationally periodic ring: $\boldsymbol{w}$ is made up of the field coefficients over the unit cell consisting of the first $p$ defect cylinders, and the field in the next unit cell can be obtained by multiplying $\boldsymbol{w}$ by the Bloch phase factor $\rho_{j}=e^{2 \pi i(j-1) / N_{e}}$, where the angular rotation by $2 \pi / N_{e}$ radians takes the place of a lattice displacement.

The solution technique for a block circulant eigenproblem provides a powerful tool for the calculation of the eigenvectors because it reduces the original spectral problem of order $N_{e} p$ to $N_{e}$ decoupled eigenproblems of order $p$. For eigenstates of defect modes arising from degenerate bands, the corresponding matrices have also blockcirculant nature, and we will next apply the method of the block circulant matrix to find the eigenstates.

\section{DEGENERATE CASE}

The treatment above involving block circulant matrices cannot be extended in its full generality to the degenerate case. There are two reasons for this: firstly, the basis of Bloch modes has more than one element so the field approximation (6) involves at least two components per 


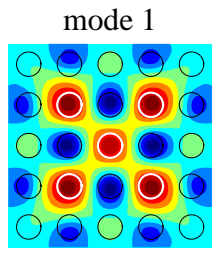

mode 4

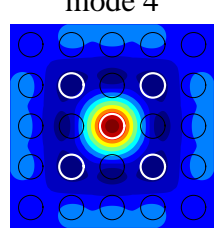

mode 2 (a)

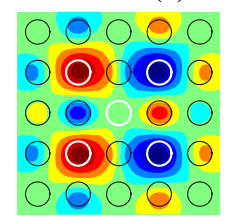

mode 5

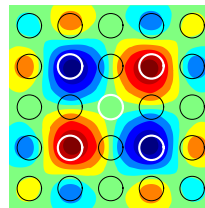

mode 2 (b)

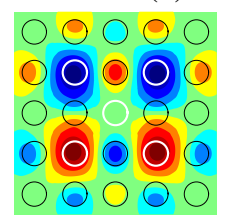

Defect cylinders
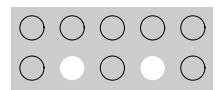

00000 00000

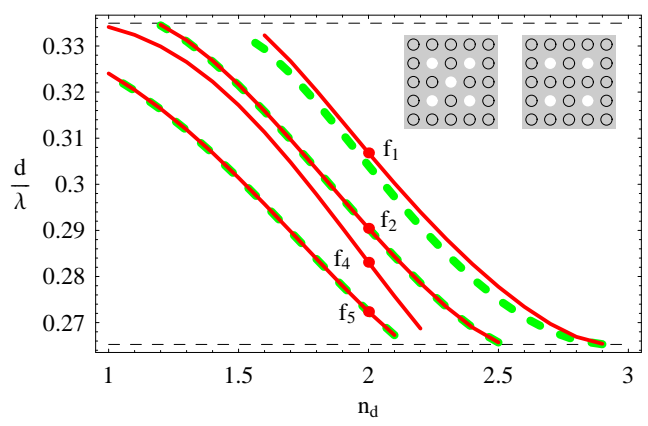

FIG. 10: (Color online) Evolution of defect arranged as a square with a centre: The top panel show the defect cylinders and the field profiles for the defect modes corresponding to $n_{d}=2$. The lower panel shows the defect dispersion curves for a square defect with centre (continuous lines) and square defects without centre (dashed line).

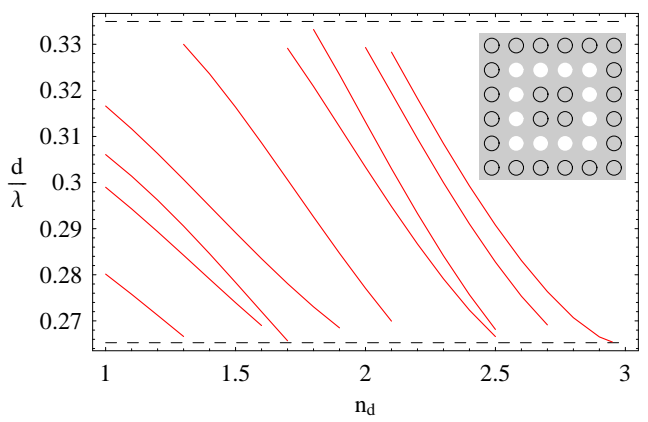

FIG. 11: (Color online) Evolution of defect arranged as a square ring.

defect cylinder $C_{j}$. Secondly, the Bloch modes on a degenerate band-edge tend to exhibit a much higher degree of anisotropy; as a consequence, the tensor $\boldsymbol{\sigma}_{L, l}$, which is appears in the envelope function $\left(K_{0} b\left|\boldsymbol{\sigma}_{L, l}^{-1}\right| \boldsymbol{r}-\boldsymbol{r}^{\prime} \mid\right.$ ) (see section II), is not generally the identity matrix. For these two reasons the matrix $\boldsymbol{M}$ of our perturbation method is not in general a circulant or block circulant matrix. However, the matrix still has a special form which is a variant of a block-circulant matrix, and so the general structure of the eigenvectors may be deduced. This analysis may be continued to determine the frequencies and degeneracies of the defect modes on a case-by-case basis. In the following, we outline the formulation of the degenerate band-edge problem.

\section{A. General formulation}

If there are $\mathcal{L}$ linearly independent Bloch modes at band-edge, then near each defect cylinder $C_{j}$, the expansion (6) becomes

$$
\psi(\boldsymbol{r}) \approx \sum_{l^{\prime}=1}^{\mathcal{L}} B_{j}^{l^{\prime}} \psi_{L}\left(\boldsymbol{k}_{L, l^{\prime}}, \boldsymbol{r}\right), \quad \boldsymbol{r} \in C_{j} .
$$

The field in Eq. (43) is written in a coordinate system with origin at the centre of the defect cylinder $C_{j}$. To derive a consistent field representation, we write Eq. (43) with respect to the cylinder $C_{1}$. We then apply the quasiperiodicity of the Bloch modes, and derive a coupling relation that is the direct analogue of Eq. (7) in the nondegenerate case, with singly degenerate Bloch mode fields replaced by linear combinations of linearly independent degenerate fields multiplied by the appropriate phase factors.

These fields are then expanded in multipole series in order to evaluate their coupling coefficients. For the examples considered in this section, the Bloch modes $\psi_{L}\left(\boldsymbol{k}_{L}, \boldsymbol{r}\right)$ at the band-edge have the symmetric multipole expansion of McIsaac [17]:

$$
\psi_{L}^{\mathcal{F}}\left(\boldsymbol{k}_{L}, \boldsymbol{r}\right)=\sum_{p=-\infty}^{\infty} b_{\nu_{L} p+m}(r) \mathcal{F}\left(\left(\nu_{L} p+m\right) \theta\right)
$$

where the symbol $\mathcal{F}$ denotes either the function cos or sin. The parameter $m \geq 0$ is an integer which characterised the azimuthal symmetry; for the modes in Fig. 13 we have $m=1$ (antisymmetry with respect to a nodal line).

As in the non-degenerate case, this leads to an eigenproblem in the form

$$
(M-I) D=0
$$

which is a generalisation of Eq. (15); $\boldsymbol{D}$ is a block vector comprising vectors $\boldsymbol{d}_{j}$, where

$$
\boldsymbol{d}_{j^{\prime}}=\sum_{l^{\prime}=1}^{\mathcal{L}}\left[\begin{array}{c}
B_{j^{\prime}}^{\left(l^{\prime}\right)} e^{i \boldsymbol{k}_{L, l^{\prime}} \cdot\left(\boldsymbol{o}_{j^{\prime}}-\boldsymbol{o}_{1}\right)} \cos \left(\left(l^{\prime}-1\right) a\right) \\
B_{j^{\prime}}^{\left(l^{\prime}\right)} e^{i \boldsymbol{k}_{L, l^{\prime}} \cdot\left(\boldsymbol{o}_{j^{\prime}}-\boldsymbol{o}_{1}\right)} \sin \left(\left(l^{\prime}-1\right) a\right)
\end{array}\right]
$$

with $a=2 \pi m / \nu_{L} . \quad M$ is a block matrix consisting of the submatrix $\boldsymbol{M}_{j, j^{\prime}}$ defined as

$$
\boldsymbol{M}_{j, j^{\prime}}=\chi \sum_{l=1}^{\nu_{L}} \beta_{j, j^{\prime}}^{(l)} \mu_{j, j^{\prime}}^{(l)}\left[\begin{array}{ll}
\cos ^{2}((l-1) a) & \frac{\sin (2(l-1) a)}{2} \\
\frac{\sin (2(l-1) a)}{2} & \sin ^{2}((l-1) a)
\end{array}\right](4
$$


where $\mu_{j, j^{\prime}}^{(l)}$ and $\chi$ are given by the definitions

$$
\mu_{j, j^{\prime}}^{(l)}=e^{i \boldsymbol{k}_{L, l} \cdot\left(\boldsymbol{o}_{j}-\boldsymbol{o}_{j^{\prime}}\right)}, \quad \chi=\frac{2 \theta_{L}}{s \mathcal{S}} \frac{\delta \mathcal{E}}{\mathcal{E}_{L}} .
$$

and $\beta_{j, j^{\prime}}^{(l)}$ is given by Eq. (11) with the difference vector $\boldsymbol{o}_{j}-\boldsymbol{o}_{j^{\prime}}$ replaced by the vector $\boldsymbol{\sigma}_{\boldsymbol{k}_{L, l}}^{-1}\left(\boldsymbol{o}_{j}-\boldsymbol{o}_{j^{\prime}}\right)$, thus taking into account the band-edge anisotropy.

For a polygonal composite defect, the matrix $M$ is generally neither circulant nor block circulant because of the high degree of anisotropy of the Bloch modes. However since the segment joining consecutive defect cylinders rotates by an angle $2 \pi / N_{e}$ as one moves from one segment to the next, by rotating the basis functions accordingly each elementary block of rows will be cyclically shifted one element to the right with respect to the preceding row. Now by applying a similarity transformation to express the problem back into the original basis, the matrix $\boldsymbol{M}$ can be written as variant of the block circulant matrix (37):

$$
\boldsymbol{M}=\left[\begin{array}{ccc}
\hat{\boldsymbol{M}}_{0} & \cdots & \hat{\boldsymbol{M}}_{N_{e}-1} \\
\boldsymbol{R} \hat{\boldsymbol{M}}_{N_{e}-1} \boldsymbol{R}^{-1} & \cdots & \boldsymbol{R} \hat{\boldsymbol{M}}_{N_{e}-2} \boldsymbol{R}^{-1} \\
\boldsymbol{R}^{2} \hat{\boldsymbol{M}}_{N_{e}-2} \boldsymbol{R}^{-2} & \cdots & \boldsymbol{R}^{2} \hat{\boldsymbol{M}}_{N_{e}-3} \boldsymbol{R}^{-2} \\
\vdots & \vdots & \vdots \\
\boldsymbol{R}^{N_{e}-1} \hat{\boldsymbol{M}}_{1} \boldsymbol{R}^{-N_{e}+1} & \cdots & \boldsymbol{R}^{N_{e}-1} \hat{\boldsymbol{M}}_{0} \boldsymbol{R}^{-N_{e}+1}
\end{array}\right]
$$

where $\hat{\boldsymbol{M}}_{j-1}=\boldsymbol{M}_{1, j}$ for $j=1, \ldots, N$ and $\boldsymbol{R}$ is the rotation transformation by an angle $2 \pi / N_{e}$.

The treatments of Eqs (38) and (39) can be generalized to the matrix $\boldsymbol{M}$ in Eq. (49). For a root of unity $\rho_{j}=e^{2 \pi i(j-1) / N_{e}}$, any eigenvalue $\xi$, with an associated eigenvector $\boldsymbol{w}$, of the matrix

$$
\boldsymbol{T}_{j}=\sum_{l=0}^{N_{e}-1} \rho_{j}^{l} \hat{\boldsymbol{M}}_{l} \boldsymbol{R}^{l}
$$

is an eigenvalue of $M$ and the corresponding eigenvector is

$$
\boldsymbol{D}=\left[\begin{array}{c}
\boldsymbol{w} \\
\rho_{j} \boldsymbol{R} \boldsymbol{w} \\
\vdots \\
\rho_{j}^{N_{e}-1} \boldsymbol{R}^{N_{e}-1} \boldsymbol{w}
\end{array}\right]
$$

Equations (50) and (51) can be obtained by adapting the derivation presented in Ref. [10] for the reduction of the eigenproblem for the block circulant matrix (37) to Eqs. (38) and (39).

The problem has been reduced to one of finding the eigenvectors $\boldsymbol{w}$ of the matrix $\boldsymbol{T}_{j}$ : once these have been determined, the structure of the defect mode is given by Eq.(51). For real-valued eigenvectors, the fields around each cylinder for successive defect modes have been rotated by an angle of $2 \pi / N_{e}$, as can be seen for the plots "mode 1" and "mode 4" in Fig. 14 or the plots "mode 3" and "mode 6" in Fig. 15.

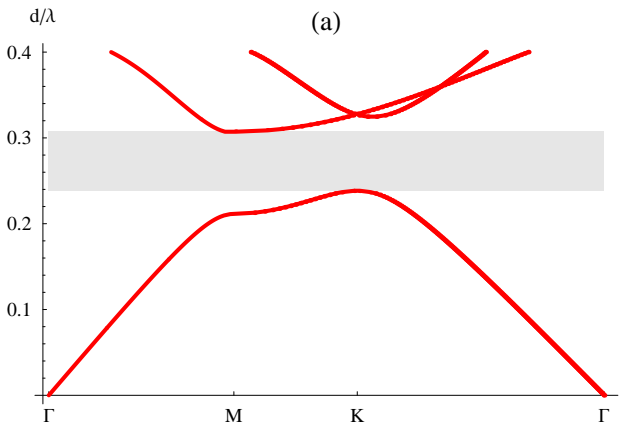

FIG. 12: (Color online) (a) Band diagram (TE polarization) for a hexagonal lattice of cylinder holes of normalized radius $a / d=0.3$ and refractive index $n_{c}=1$ in a dense background of refractive index $n_{b}=3$.

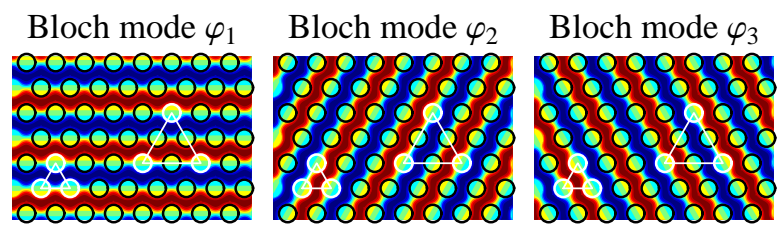

FIG. 13: (Color online) Bloch modes: The three plots show the Bloch modes $\psi_{1}=\psi_{L}\left(\boldsymbol{k}_{L, 1}, \boldsymbol{r}\right) \psi_{2}=\psi_{L}\left(\boldsymbol{k}_{L, 2}, \boldsymbol{r}\right)$, and $\psi_{3}=\psi_{L}\left(\boldsymbol{k}_{L, 3}, \boldsymbol{r}\right)$ at upper edge ( $M$ point of the Brillouin zone) of the first gap of the PC described in Fig. 12. The components of the wave vectors are $\boldsymbol{k}_{L, 1}=[0,2 \pi /(d \sqrt{3})]^{T}$, $\boldsymbol{k}_{L, 2}=\boldsymbol{R} \boldsymbol{k}_{L, 1}$ and $\boldsymbol{k}_{L, 3}=\boldsymbol{R}^{2} \boldsymbol{k}_{L, 1}$ where the $\boldsymbol{R}$ denotes the matrix corresponding to a rotation by $\pi / 3$.

\section{B. Example of an equilateral arrangement of defects}

We now give an example of the calculation of a defect state near a degenerate band of a hexagonal array. We consider a $\mathrm{PC}$ with a hexagonal array of holes operated in TE polarization. The holes have refractive index $n_{c}=1$ and normalized radius $a / d=0.3$, while the background has refractive index $n_{b}=3$. As shown in Fig. 12, the structure has a band gap for $d / \lambda \in[0.23849,0.30719]$, the upper edge of which occurs at the $M$ point of the Brillouin zone. There are three linearly independent Bloch modes at the band-edge corresponding to the $M$ point; they are associated with the wave vectors $\boldsymbol{k}_{L, 1}=[0,2 \pi /(d \sqrt{3})]^{T}, \boldsymbol{k}_{L, 2}=\boldsymbol{R}_{\nu_{L}} \boldsymbol{k}_{L, 1}$ and $\boldsymbol{k}_{L, 3}=\boldsymbol{R}_{\nu_{L}}^{2} \boldsymbol{k}_{L, 1}$ where $\boldsymbol{R}_{\nu_{L}}$ denotes the rotation by an angle $2 \pi / \nu_{l}=\pi / 3$. Figure 13 shows the Bloch modes $\psi_{L}\left(\boldsymbol{k}_{L, 1}, \boldsymbol{r}\right), \psi_{L}\left(\boldsymbol{k}_{L, 2}, \boldsymbol{r}\right)$ and $\psi_{L}\left(\boldsymbol{k}_{L, 3}, \boldsymbol{r}\right)$ while the white cylinders illustrate two equilateral triangles which are considered below. We observe that the phases of each Bloch mode are the same for the three cylinders centred at the vertices of the triangles with side length $2 d$, which means that $\mu_{j, j^{\prime}}^{(l)}=1$ for all modes $\psi_{L}\left(\boldsymbol{k}_{L, l}, \boldsymbol{r}\right)$ and all cylinders $C_{j}$ and $C_{j^{\prime}}$. 
A composite defect is created by increasing the refractive index of the cylinders $C_{1}, C_{2}$, and $C_{3}$ from $n_{c}=1$ to a value $n_{d}>n_{c}$. Since the perturbation is positive, i.e., $\delta \mathcal{E}>0$, defect modes emerge from the upper gap edge. The cylinders $C_{1}, C_{2}$, and $C_{3}$ are arranged as an equilateral triangle and are centred respectively at $\boldsymbol{o}_{1}=(0,0)$, $\boldsymbol{o}_{2}=h d(1,0)$ and $\boldsymbol{o}_{3}=h d(1, \sqrt{3}) / 2$ where $h$ is an integer which represents the normalized side length of the triangle. Figures 14 and 15 show the defect dispersion curves with $h=1$ and $h=2$ respectively. We can see that, with $h=1$, there are two distinct curves which approach the upper gap edge as $\delta \mathcal{E}$ tends to zero, i.e., as $n_{d}$ tends to $n_{c}=3$, while with $h=2$ there is only one such curve.

Our theory can explain this behaviour of the fundamental mode. We recall that, in the case of nondegenerate band-edge, we determined the fundamental mode of a polygonal defect by substituting the approximation $\beta_{j, j^{\prime}}^{(l)} \approx \ln |\delta \omega| / 2$ into the expressions for the eigenvalues $\xi$. When the substitution of the leading order term $\ln |\delta \omega| / 2$ gives a nonzero value then the corresponding eigenvalue has the logarithmic singularity $\ln |\delta \omega|$ and, consequently, it is associated with a fundamental mode. However, with the degenerate band-edge, the eigenvalue expressions are usually complicated nonlinear formulas. We first substitute the leading order estimate $\beta_{j, j^{\prime}}^{(l)} \approx \ln |\delta \omega| / 2$ into the expressions (47) for the matrix coefficients

$$
\begin{aligned}
& \hat{\boldsymbol{M}}_{0}=\frac{3}{2} \chi \ln |\delta \omega|\left[\begin{array}{ll}
1 & 0 \\
0 & 1
\end{array}\right] \\
& \hat{\boldsymbol{M}}_{1}=\frac{1}{2} \chi \ln |\delta \omega|\left[\begin{array}{cc}
2+(-1)^{h} & 0 \\
0 & 3(-1)^{h}
\end{array}\right] \\
& \hat{\boldsymbol{M}}_{2}=\frac{1}{4} \chi \ln |\delta \omega|\left[\begin{array}{cc}
1+5(-1)^{h} & \left(1-(-1)^{h}\right) \sqrt{3} \\
\left(1-(-1)^{h}\right) \sqrt{3} & 3+3(-1)^{h}
\end{array}\right] .
\end{aligned}
$$

We then study the eigenvalues of these matrices for the specific geometrical structure of the composite defect.

When the normalized side length of the composite defect is even, we have

$$
\hat{\boldsymbol{M}}_{0}=\hat{\boldsymbol{M}}_{1}=\hat{\boldsymbol{M}}_{2}=\frac{3}{2} \chi \ln |\delta \omega|\left[\begin{array}{ll}
1 & 0 \\
0 & 1
\end{array}\right] .
$$

The matrices $\hat{\boldsymbol{M}}_{l} \boldsymbol{R}^{l}$ used to construct $\boldsymbol{T}_{j}$ are

$$
\begin{aligned}
\hat{\boldsymbol{M}}_{0} \boldsymbol{R}^{0} & =\frac{3}{2} \chi \ln |\delta \omega|\left[\begin{array}{ll}
1 & 0 \\
0 & 1
\end{array}\right], \\
\hat{\boldsymbol{M}}_{1} \boldsymbol{R} & =\frac{3}{4} \chi \ln |\delta \omega|\left[\begin{array}{cc}
-1 & -\sqrt{3} \\
\sqrt{3} & -1
\end{array}\right], \\
\hat{\boldsymbol{M}}_{2} \boldsymbol{R}^{2} & =\frac{3}{4} \chi \ln |\delta \omega|\left[\begin{array}{cc}
-1 & \sqrt{3} \\
-\sqrt{3} & -1
\end{array}\right] .
\end{aligned}
$$

We now examine the eigenvalues of $\boldsymbol{T}_{j}$ in Eq. (50) for $\rho_{1}=1, \rho_{2}=e^{2 \pi i / 3}$ and $\rho_{3}=\rho_{2}^{*}$. For $\rho_{1}=1$, we have $\boldsymbol{T}_{1}=\mathbf{0}$ implying that $\boldsymbol{T}_{1}$ has no nonzero eigenvalue. Thus there is no fundamental mode associated with $\boldsymbol{T}_{1}$.
With $\rho_{2}=e^{2 \pi i / 3}$, we have

$$
\begin{aligned}
\boldsymbol{T}_{2} & =\frac{9}{4} \chi \ln |\delta \omega|\left[\begin{array}{cc}
1 & -i \\
i & 1
\end{array}\right] \\
& =\frac{9}{4} \chi \ln |\delta \omega|\left[\begin{array}{cc}
1 & 1 \\
i & -i
\end{array}\right]\left[\begin{array}{ll}
2 & 0 \\
0 & 0
\end{array}\right]\left[\begin{array}{cc}
1 & 1 \\
i & -i
\end{array}\right]^{-1}
\end{aligned}
$$

Thus $\boldsymbol{T}_{2}$ has only one nonzero eigenvalue $\xi=$ $9 \chi \ln |\delta \omega| / 2$ and it is associated with an eigenvector $\boldsymbol{w}_{1}=[1, i]^{T} ; \xi$ is also an eigenvalue of $\boldsymbol{M}$ which is associated with the eigenstate given by Eq. (51). We note that $\boldsymbol{w}_{1}=[1, i]^{T}$ is also an eigenvector $\boldsymbol{R}$ associated with an eigenvalue equal to $\rho_{2}^{*}$ and by substituting the relation $\rho_{2}^{l} \boldsymbol{R}^{l} \boldsymbol{w}_{1}=\left(\rho_{2} \rho_{2}^{*}\right)^{l} \boldsymbol{w}_{1}=\boldsymbol{w}_{1}$, into Eq. (51), we see that the eigenvector of $\boldsymbol{M}$ associated with the eigenvalue $\xi$ takes the form $\boldsymbol{D}=\left[\boldsymbol{w}_{1}^{T}, \ldots, \boldsymbol{w}_{1}^{T}\right]^{T}$.

In a similar way, the root $\rho_{3}=\rho_{2}^{*}$ generates one nonzero eigenvalue $\xi=9 \chi \ln |\delta \omega| / 2$ of $\boldsymbol{T}_{3}$ associated with an eigenvector $\boldsymbol{w}_{2}=[1,-i]^{T} ; \xi$ is also an eigenvalue of $\boldsymbol{M}$ which is associated with $\boldsymbol{D}=\left[\boldsymbol{w}_{2}^{T}, \ldots, \boldsymbol{w}_{2}^{T}\right]^{T}$.

If the side length is odd, the matrices $\hat{M}_{l} \boldsymbol{R}^{l}$ are

$$
\begin{aligned}
\hat{\boldsymbol{M}}_{0} \boldsymbol{R}^{0} & =\frac{1}{2} \chi \ln |\delta \omega|\left[\begin{array}{ll}
3 & 0 \\
0 & 3
\end{array}\right], \\
\hat{\boldsymbol{M}}_{1} \boldsymbol{R} & =\frac{1}{4} \chi \ln |\delta \omega|\left[\begin{array}{cc}
-1 & -\sqrt{3} \\
-3 \sqrt{3} & 3
\end{array}\right], \\
\hat{\boldsymbol{M}}_{2} \boldsymbol{R}^{2} & =\frac{1}{4} \chi \ln |\delta \omega|\left[\begin{array}{cc}
-1 & -3 \sqrt{3} \\
-\sqrt{3} & 3
\end{array}\right]
\end{aligned}
$$

We now examine the eigenvalues of $\boldsymbol{T}_{j}$. For $\rho_{1}=1$, we have

$$
\begin{aligned}
\boldsymbol{T}_{1} & =\chi \ln |\delta \omega|\left[\begin{array}{cc}
1 & -\sqrt{3} \\
-\sqrt{3} & 3
\end{array}\right] \\
& =\chi \ln |\delta \omega| \boldsymbol{R}\left[\begin{array}{ll}
4 & 0 \\
0 & 0
\end{array}\right] \boldsymbol{R}^{-1}
\end{aligned}
$$

so that $\boldsymbol{T}_{1}$ has a simple nonzero eigenvalue $\xi=4 \chi \ln |\delta \omega|$ which is associated with an eigenvector $\boldsymbol{w}=[1,-\sqrt{3}]^{T}$.

A similar calculation with $\rho_{2}=e^{2 \pi i / 3}$ gives a nonzero eigenvalue $\xi=5 \chi \ln |\delta \omega| / 2$ of $\boldsymbol{T}_{2}$ which is associated with an eigenvector $\boldsymbol{w}=[-i+2 / \sqrt{3}, 1]^{T}$. Since $\rho_{3}=\rho_{2}^{*}$, the matrix $\boldsymbol{T}_{3}$ has the same eigenvalue as $\boldsymbol{T}_{2}$ while its eigenvectors are conjugate to those of $\boldsymbol{T}_{2}$; this means that the fundamental mode corresponding to the conjugate pair $\rho_{2}$ and $\rho_{3}$ is doubly degenerate.

In each case, the fundamental eigenvalue has the form $\xi=\eta \chi \ln |\delta \omega|+\ln \mathcal{B}$ with $\ln \mathcal{B}=O\left(|\delta \omega|^{0}\right)$; we can then show that, to leading order term, the solution of dispersion equation $\xi(\delta \omega)=1$ exhibits an exponential behaviour near the gap edge. Indeed, from the definition (48) and the fact that $\theta_{L}=1 / 2$ (the $M$ point lies at a mid-edge of the Brillouin zone) and $s=1$ (maximum of a band surface) the solution $\xi(\delta \omega)=1$ take the form

$$
\delta \omega=\mathcal{B} \exp \left(\frac{\mathcal{S} \mathcal{E}_{L}}{\eta \delta \mathcal{E}}\right)
$$


which is analogue to Eq. (24) for the fundamental of a double defect near a nondegenerate band edge. For the TE polarization, the mode field is represented by the component $H_{z}$ and the change in the relative electric energy is

$$
\begin{aligned}
\frac{\delta \mathcal{E}}{\mathcal{E}_{L}} & =\frac{\int_{C_{1}} \delta \varepsilon(\boldsymbol{r})\left\|\boldsymbol{E}_{L}\left(\boldsymbol{k}_{L}, \boldsymbol{r}\right)\right\|^{2} d^{2} \boldsymbol{r}}{\int_{\mathrm{WSC}} \varepsilon(\boldsymbol{r})\left\|\boldsymbol{E}_{L}\left(\boldsymbol{k}_{L}, \boldsymbol{r}\right)\right\|^{2} d^{2} \boldsymbol{r}}, \\
& =\frac{\left(n_{d}^{2}-n_{c}^{2}\right) \int_{C_{1}}\left\|\nabla \psi_{L}\left(\boldsymbol{k}_{L}, \boldsymbol{r}\right)\right\|^{2} d^{2} \boldsymbol{r}}{n_{c}^{4} \int_{\mathrm{WSC}} \varepsilon(\boldsymbol{r})^{-1}\left\|\nabla \psi_{L}\left(\boldsymbol{k}_{L}, \boldsymbol{r}\right)\right\|^{2} d^{2} \boldsymbol{r}} .
\end{aligned}
$$

As we show above, $\eta=9 / 2$ for the fundamental mode for the example in Fig. 15 while the two curves of fundamental modes in Fig. 14 we have $\eta=4$ and $\eta=5 / 2$. For a comparison, with the degenerate band edge considered here, the fundamental mode of a single cylinder defect follows an exponential with a factor $\eta=3 / 2$ [8]. We have determined the prefactor $\mathcal{B}$ in Eq. (66) by fitting the defect dispersion curve of the fundamental mode to the exponential law Eq. (66). Figure 16 shows that there is a good agreement between the defect dispersion curve and the fitted exponential law.

Thus for a defect cluster arranged as an equilateral triangle with an odd normalized side length, the fundamental modes consist of two dispersion curve curves: one curve of doubly degenerate modes and one curve of rotationally invariant nondegenerate modes (see Fig. (14)). This is in contrast to the even side length case where there is only a degenerate fundamental mode.

\section{DISCUSSION AND CONCLUSION}

We have analysed defect modes in $2 \mathrm{D}$ photonic crystals in both TE and TM polarisations. The generality of the theory in $2 \mathrm{D}$, which is based on symmetry, is a strong indication of its applicability to 3D photonic crystal slabs. We also note that the work presented here reproduces the results presented by McIsaac [17] in the context of optical fibres for defect states possessing the same symmetry as that of the lattice.

The work here shows a similarity between defect modes and the modes of waveguides with a two-dimensional cross-section: for example, there is always at least a single defect mode and when the defect gets stronger, more modes are drawn in. A difference between the two geometries is that in the waveguide problem fields outside the guide decays exponentially but does not change sign. Here, because the fields are linked to the Bloch functions of the underlying lattice, the situation is different: the evanescently decaying field can change sign leading to a reordering of the modes in some situations.

A central aim of the work presented in this paper is to deduce as much information as possible about the structure of the eigenstates and eigenvalues of the composite defect from the physical arrangement of the defect sites. It is a natural consequence that the most general rules may be deduced when both the physical structure of the

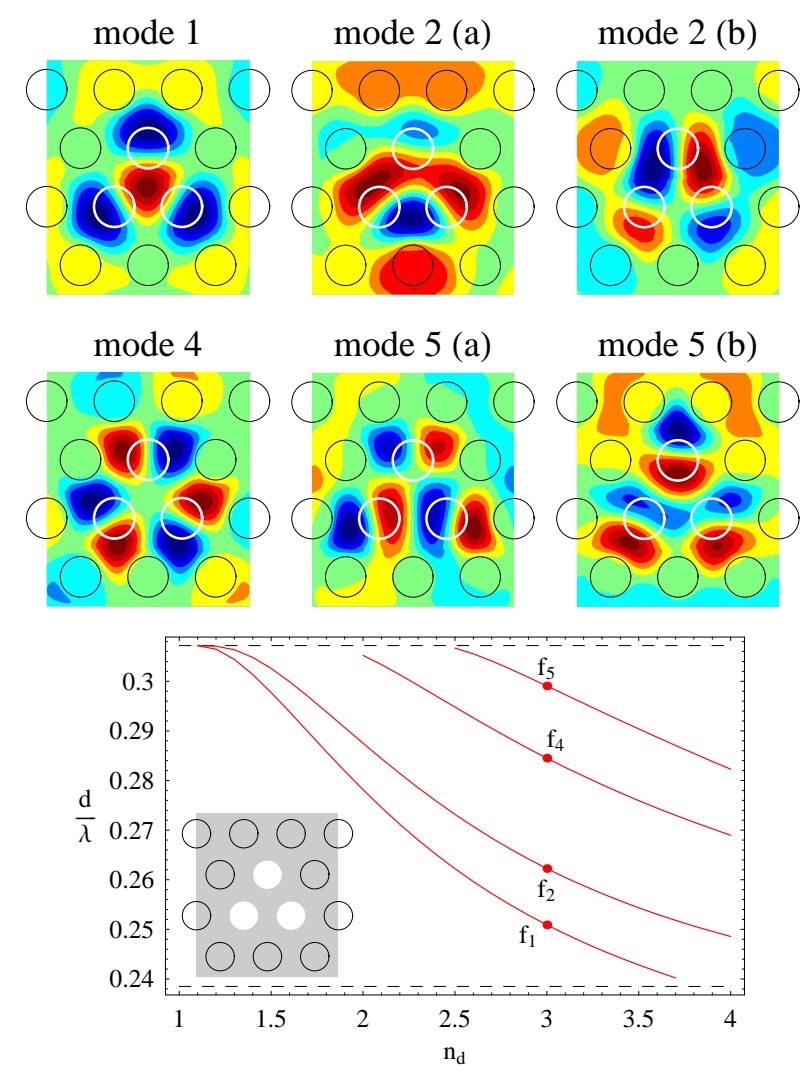

FIG. 14: (Color online) Evolution of the state associated with a defect arranged as an equilateral triangle of normalized side length $h=1$. The defect is created in the PC described in Fig. 12. The fundamental modes exist on two curves. As the field plots show one the fundamental modes are nondegenerate on the curve containing the point $f_{1}$ and doubly degenerate on the curve containing $f_{2}$.

composite defect and the Bloch modes on the band edge possess a high degree of symmetry. We have found that as this symmetry is broken the treatment becomes more complex, in the sense that the eigenvectors depend on additional parameters appearing in the problem. Thus, the treatment presented here works particularly elegantly for non-degenerate band-edges for which the defect sites are in geometrically equivalent positions, to within a lattice rotation. This leads to a circulant coupling matrix, and in these cases the eigenvectors are determined independent of coupling strength and distance between the defects. Although such a high degree of symmetry does not exist for rotationally symmetric compound defects arranged in a ring, we have seen that the same formalism may still be applied: the eigenvectors are made up a set of eigensolutions to subelements of the composite defect, and the structure of these subelements in the total eigensolution is again independent of the coupling strength. Finally, for degenerate band-edges, not only does the eigensolution depend on a set of subelements of the problem, but the relation between the subelements 

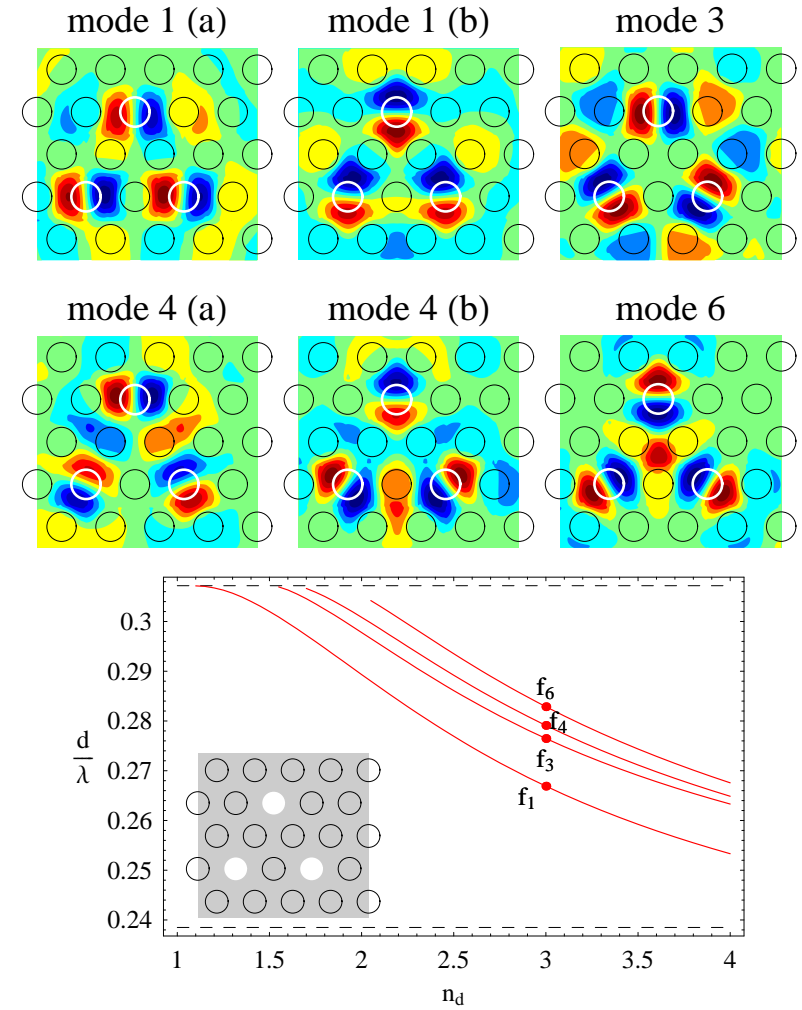

FIG. 15: (Color online) Evolution of defect arranged as an equilateral triangle of normalized side length $h=2$. The fundamental modes are degenerate and correspond to the curve containing the point $f_{1}$.
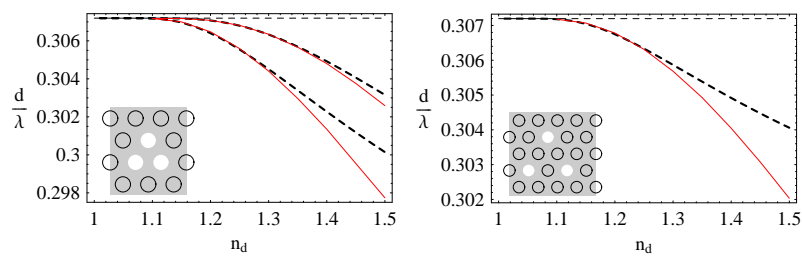

FIG. 16: (Color online) Evolution of defect arranged as an equilateral triangle: The continuous curve shows the defect mode frequency from the FSS method while the dashed black curve is a fit of the exponential law Eq. (66). depends on the composite defect geometry and on the relative phases of the Bloch modes on the band edge. Although it is then more difficult to make predictions about the eigenvectors in general, the problem may be reduced to a set of simple subproblems in each particular case, and the overall structure of the eigensolution can then be determined. One of the motivations for the present work is that the multiplicity of modes arising from composite defect states can cause confusion when a brute-force computation is attempted: one may experience difficulty in distinguishing real states from numerical artifacts, distinguishing one state from another, or even determining the number and degeneracy of the states resulting from a given type of perturbation. The approach presented here helps resolve these issues. In addition, the first-order asymptotic approximation given here is potentially very useful for determining not only the band-edge fields but also in giving an accurate estimate for their frequencies.

Apart from the study of defects for their own sake, the methods presented here can be used for the design of resonant structures in photonic crystal devices. In order to design, for instance, an add-drop filter using a composite defect state in a photonic crystal structure, one must know not only the frequencies of the individual states but also the phase relationship between the defect sites in the resonator. The ready calculation and classification of composite defect states can be used to gain insight into the link between the set of resonances in photonic crystal ring resonators and the dynamics of pulse propagation in such structures: one expects that the pulse dispersion and group velocity depend intimately on the phase relationship of the set of resonant states. Finally, the knowledge of the eigenvectors and corresponding resonant frequencies may be used for efficient field expansions.

\section{Acknowledgements}

This work was produced with the assistance of the Australian Research Council under the ARC Centres of Excellence program.
[1] Z. Qiang, W. Zhou, and R. A. Soref, Opt. Express 15, 1823 (2007).

[2] W.-Y. Chiu, T.-W. Huang, Y.-H. Wu, Y.-J. Chan, C.-H. H. H.-T. Chien, and C.-C. Chen, Opt. Express 15, 15500 (2007).

[3] K. Ogusu and K. Takayama, Opt. Express 16, 7525 (2008).

[4] S.-H. Kim, H.-Y. Ryu, H.-G. Park, G.-H. Kim, Y.-S. Choi, Y.-H. Lee, and J.-S. Kim, Appl. Phys. Lett. 81, 2499 (2002).

[5] A. R. Alija, L. J. Martínez, P. A. Postigo, C. Seassal, and
P. Viktorovitch, Appl. Phys. Lett. 89, 101102 (2006).

[6] S. G. Johnson, M. Ibanescu, M. A. Skorobogatiy, O. Weisberg, J. D. Joannopoulos, and Y. Fink, Phys. Rev. E 65, 066611 (2002).

[7] M. L. Povinelli, S. G. Johnson, E. Lidorikis, J. D. Joannopoulos, and M. Soljačić, Appl. Phys. Lett. 84, 3639 (2004).

[8] K. B. Dossou, L. C. Botten, R. C. McPhedran, C. G. Poulton, A. A. Asatryan, and C. M. de Sterke, Phys. Rev. A 77, 063839 (2008).

[9] B. Friedman, Proc. Cambridge Philos. Soc. 57, 37 (1961). 
[10] G. J. Tee, Res. Lett. Inf. Math. Sci 8, 123 (2005), URL www.massey.ac.nz/ wwiims/research/letters.

[11] P. J. Davis, Circulant matrices (John Wiley \& Sons, New York, 1979).

[12] S. Mahmoodian, R. C. McPhedran, C. M. de Sterke, K. B. Dossou, C. G. Poulton, and L. C. Botten, Phys. Rev. A 79, 013814 (2009).

[13] S. Wilcox, L. C. Botten, R. C. McPhedran, C. G. Poulton, and C. M. de Sterke, Phys. Rev. E 71, 056606 (2005).

[14] L. C. Botten, K. B. Dossou, S. Wilcox, R. C. McPhedran,
C. M. de Sterke, N. A. Nicorovici, and A. A. Asatryan, Int. J. Microwave and Optical Technology 1, 133 (2006).

[15] K. B. Dossou, L. C. Botten, S. Wilcox, R. C. McPhedran, C. M. de Sterke, N. A. Nicorovici, and A. A. Asatryan, Physica B 394, 330 (2007).

[16] C. M. de Sterke, L. C. Botten, A. A. Asatryan, T. P. White, and R. C. McPhedran, Opt. Lett. 29, 1384 (2004).

[17] P. McIsaac, IEEE Trans. Microwave Theory Tech. 23, $421(1975)$. 